\title{
Technische Hochschulen:
}

Attraktive Arbeitsorte für Frauen und

Männer?

Der Weg von technischen Hochschulen

zu geschlechtergerechten Organisationen

\author{
Antje Ducki \\ Randi Worath \\ Hedda Ofoole Knoll \\ Lena Ziesmann
}

Schriftenreihe des Gender- und Technikzentrum (GuTZ)

der Beuth Hochschule für Technik Berlin

Band 12

Herausgeberinnen

Eva-Maria Dombrowski, Antje Ducki 
(C) 2020 Dieses Werk ist im Verlag Barbara Budrich erschienen und steht unter folgender Creative Commons Lizenz:

https://creativecommons.org/licenses/by-sa/4.0/legalcode

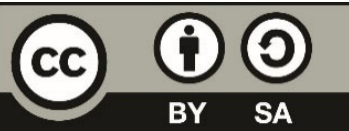

Verbreitung, Speicherung und Vervielfältigung erlaubt, kommerzielle Nutzung und Veränderung nur mit Genehmigung des Verlags Budrich Academic.

Dieses Werk steht im Open Access Bereich der Verlagsseite zum kostenlosen Download bereit (https://doi.org/10.3224/96665988).

eISBN 978-3-96665-988-8 (eBook)

DOI 10.3224/96665988

\section{Verlag Budrich Academic Press}

http://www.budrich-academic.de 
Prof. in Dr. in Antje Ducki ist Professorin für Arbeits- und Organisationspsychologie an der Beuth Hochschule für Technik Berlin. Zusammen mit Prof. Dr. Eva Maria Dombrowski leitet sie seit 2009 das Gender- und Technik-Zentrum (GuTZ). Im Mittelpunkt ihrer Forschungsarbeiten stehen die Themen betriebliche Gesundheitsförderung, Mobilität und Gesundheit sowie verschiedene Gender- und Diversity-Themen.

Randi Worath ist Referentin für Berufungen im Präsidium der Beuth Hochschule für Technik Berlin. Zuvor war sie wissenschaftliche Mitarbeiterin für die Themen Personalmanagement an Hochschulen, Diversity Sensibilisierung und Gender. Vor ihrem Wechsel an die Hochschule arbeitete sie als Abteilungsleiterin im Qualitätsmanagement im Maschinenbau.

Dr. in Hedda Ofoole Knoll ist als wissenschaftliche Mitarbeiterin (50\%-Stelle) an der Beuth Hochschule für Technik Berlin in dem geförderten BCP-Projekt „Geschlechtergerechte Berufungsbedingungen“ tätig. Im Mittelpunkt ihrer Arbeit stehen die Themen Gender und Diversity im Personalmanagement an deutschen Hochschulen. Zusätzlich arbeitet sie als „Head of Belonging and Anti-Discrimination in employer relations management“ bei der Firma Tbd*.

Lena Ziesmann leitet seit 2017 das Referat Qualitätsmanagement an der Beuth Hochschule für Technik Berlin. Schwerpunkt Ihrer Arbeit ist die Weiterentwicklung der Qualität der Lehre und der Studiengänge der Beuth Hochschule. Als Expertin für Umfrage- und insbesondere Evaluationsforschung ist Sie unter anderem für die Evaluation der Lehre aber auch für hochschulweite Untersuchungen der Studierenden und Lehrenden verantwortlich.

Gefördert durch das

\section{$\mathrm{BCP}$}

Chancengleichheitsprogramm

Diese Publikation wurde über das Berliner Programm zur Förderung der Chancengleichheit für Frauen in Forschung und Lehre (BCP) gefördert.

Impressum

Herausgeberinnen:

Prof. in Dr. in Eva-Maria Dombrowski

Prof. in Dr. in Antje Ducki

Redaktion: Gender- und Technik-Zentrum der Beuth Hochschule für Technik

Luxemburger Str. 10

13353 Berlin

E- Mail: gutz@beuth-hochschule.de

Internet: https://www.beuth-hochschule.de/gutz/

Verantwortlich für den Inhalt sind die Autor*innen 


\section{Inhaltsverzeichnis}

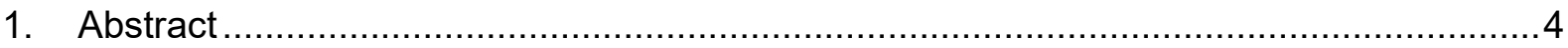

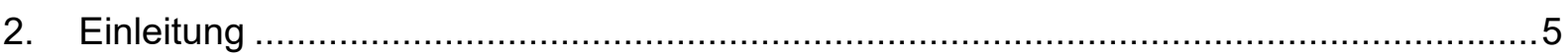

3. Frauen in der Wissenschaft - Anspruch und Realität .......................................... 6

4. Karrieren an Hochschulen für angewandte Wissenschaften .....................................8

5. Wirkmächtige Geschlechterstereotypien.............................................................. 11

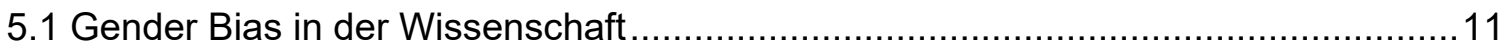

5.2 Technische Fachkulturen - Frau ist ein ,Token', ob sie will oder nicht .................... 13

6. Nutzen und Funktion von Organisationklimaanalysen .............................................15

7. Organisationsklimaanalyse an der Beuth Hochschule für Technik .............................16

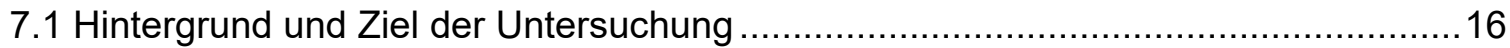

7.2 Inhalt, Aufbau und Durchführung der Untersuchung...........................................17

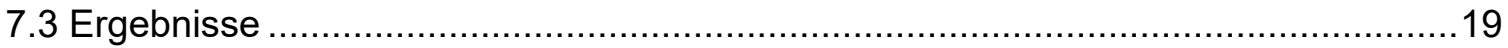

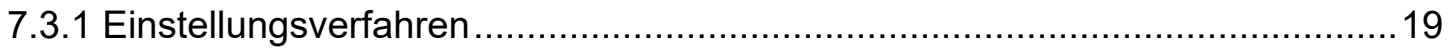

7.3.2 Entscheidungsfaktoren, die zur Bewerbung geführt haben..........................21

7.3.3 Berufliche Tätigkeiten (Verteilung der Arbeitszeit, verfügbare Ressourcen) ....22

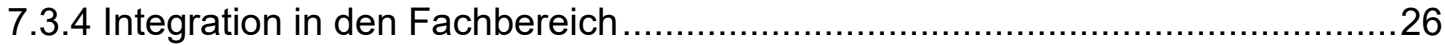

7.3.5 Fachbereichsmaßnahmen zur Gewinnung von Frauen in Professuren ...........28

7.3.6 Programme und Ressourcen an der Beuth Hochschule für Technik ...............30

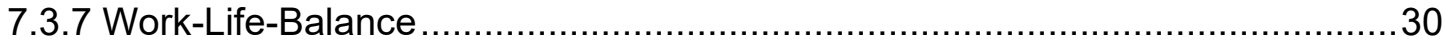

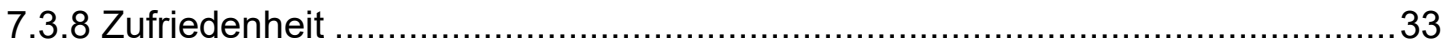

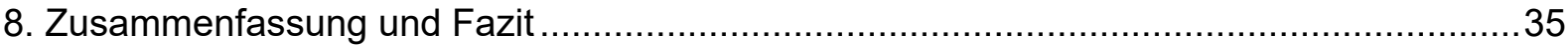

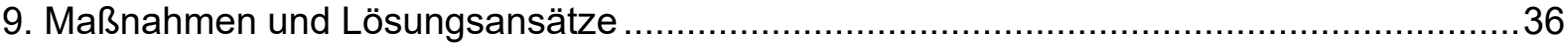

Abbildungen …

Tabellen

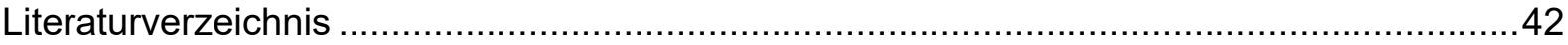

Bisher erschienene Bände der Schriftenreihe des GuTZ ..................................................47 


\section{Abstract}

Die Partizipation von Frauen an Professuren ist in den letzten Jahren gestiegen, dennoch sind Professorinnen im Wissenschaftssystem nach wie vor unterrepräsentiert. Diese geringe Repräsentanz von Professorinnen im Wissenschaftssystem wird seit langem erforscht. Studien, insbesondere über die Karrierewege von Professorinnen an technischen Hochschulen sind selten, Ergebnisse entsprechend wenig evidenzbasiert. Mit dieser Studie1 soll exemplarisch für eine technische Hochschule, der Frage nachgegangen werden, welche Aspekte für eine geschlechtergerechte Hochschulkultur relevant sind und ob diese Ansatzpunkte liefern können, um den Frauenanteil an Professuren insgesamt, aber insbesondere in den MINT-Disziplinen (gemeint sind Mathematik, Informatik, Naturwissenschaft und Technik) zu verstärken.

Hierfür wurde eine Organisationsklimaanalyse an der Beuth Hochschule für Technik Berlin durchgeführt und geschlechterdifferenziert ausgewertet. Die Befragung hat neben allgemeinen Ergebnissen zu verschiedenen Aspekten des Organisationsklimas, erste Hinweise darauf gegeben, welche Maßnahmen hilfreich sein können, um mehr Frauen als Professor*innen zu gewinnen. Dabei zeigte sich, dass Professorinnen und Professoren viele Aspekte des Organisationsklimas ähnlich einschätzen. Es konnten jedoch auch Unterschiede festgestellt werden. So ist es den befragten Professorinnen im Vergleich zu den Professoren wichtiger zu forschen und sie geben häufiger an, aufgrund ihrer persönlichen Verpflichtungen auf berufliche Aktivitäten verzichten zu müssen. Gleichzeitig waren sie aber nicht der Meinung, dass die sogenannten Care-Aufgaben sie in ihrer Karriereentwicklung behindert hätten.

Auch wenn die Studie aufgrund der kleinen Stichprobe nicht repräsentativ ist, hat sie zu aufschlussreichen Ergebnissen und ersten Anregungen geführt, wie geschlechterspezifische Diskriminierung abgebaut und eine geschlechtergerechte Hochschulorganisation aufgebaut werden kann.

\section{Schlüsselwörter}

Geschlechtergerechte technische Hochschule, Unterrepräsentanz von Professorinnen, diskriminierungsfreie Hochschule, Berufungsverfahren, Karrierewege, geschlechtergerechte Organisationsmerkmale, Organisationsklimaanalyse, Arbeitszufriedenheit

\footnotetext{
${ }^{1}$ Die Studie wurde im Rahmen des Projektes „Geschlechtergerechte Berufungsbedingungen“ durchgeführt und vom Berliner Programm zur Förderung der Chancengleichheit für Frauen in Forschung und Lehre (BCP) gefördert.
} 


\section{Einleitung}

Seit einigen Jahren werden verschiedene Konzepte und Maßnahmen entwickelt und erprobt, die darauf ausgerichtet sind, Hochschulen geschlechtergerecht zu gestalten. Dabei sind die Maßnahmen in der deutschen Hochschullandschaft institutionell unterschiedlich verankert und decken ein breites Angebotsspektrum ab. Sie reichen von individuellen Unterstützungsangeboten wie Mentoring, Coaching, Karriereberatung, Stipendien und Dual Career-Angeboten bis zu strukturellen Maßnahmen, wie vorgezogene Nachfolgeberufungen oder die Bindung von Forschungsfördermitteln an stichhaltige Gleichstellungskonzepte (Leicht-Scholten \& Wolffram 2010; Devine et al. 2017; Sembritzki \& Thiele 2019). Gleichstellungsbeauftragte und -referate sind zuständig für Gleichstellungs- und Frauenfördermaßnahmen, die sich auf alle Hochschulprozesse beziehen, sowie auf die Überprüfung geschlechtergerechter Berufungsverfahren (BMJ 2012). Einige Hochschulen haben damit begonnen, gezielt Stabstellen einzurichten, um die Themen Geschlechtergerechtigkeit und Diversity in den verschiedenen Ebenen der Verwaltungshierarchien nachhaltig zu verankern (Stark \& Kiendl 2013).

Trotz der zahlreichen gleichstellungspolitischen Bemühungen und strukturellen Veränderungen an Hochschulen, sind Frauen in Professuren nach wie vor unterrepräsentiert, speziell in den MINT- Fächern (Rheinländer 2015; Sembritzki \& Thiele 2019). Zu den Ursachen dieser Unterrepräsentanz wird seit Jahrzehnten geforscht und Zahlen werden kontinuierlich berichtet (Stark \& Kiendl 2013; Van den Brink 2015; Sembritzki \& Thiele 2019). Gleichwohl existiert ein Mangel an theoriebasierter empirischer Forschung, die das Geschlecht nicht als (statistische) Unterscheidungskategorie, sondern als soziale Strukturkategorie verwendet, um Geschlechterbeziehungen, vertikale und horizontale Segregationsprozesse an Hochschulen zu analysieren und Wirkmechanismen verstehbar zu machen (Winter \& Krempkow 2013; Kunadt et al. 2014; Müller et al. 2015). Nur wenige Studien untersuchen in diesem Zusammenhang die Konstruktion von Stereotypen und ihre Wirkungen auf Handlungsmuster und Prozesse in Organisationen sowie auf die Entwicklung weiblicher und männlicher Identität (Kunadt et al. 2014; Braun et al 2015; Sembritzki \& Thiele 2019).

Hinzu kommt, dass bisherige Analysen von Karrierewegen hin zu einer Professur auf Universitäten fokussiert sind (Sembritzki \& Thiele 2019). Studien zu Karrierewegen in angewandten Hochschulen, speziell technisch angewandten Hochschulen, sind die Ausnahme (Schlegel 2006; Devine et al. 2017; Sembritzki \& Thiele 2019). Noch seltener fokussieren Studien organisatorische und strukturelle Gegebenheiten technischer Hochschulen mit Bezug zum Thema Gendergerechtigkeit (Leicht-Scholten \& Wolffram 2010; Wissenschaftsrat 2018). Auch die umfangreichen Studien zu Berufungsverfahren von Frauen (Junghans 2012; Kortendiek et al. 2014; Färber \& Riedler 2016) liefern wenig dezidierte Befunde zu den Karrierewegen in Hochschulen für angewandte Wissenschaften (HAW-Professur) (Sembritzki \& Thiele 2019). 
Diesen Fragen widmet sich die vorliegende Studie. Im Rahmen des Projektes „Geschlechtergerechte Berufungsbedingungen“, gefördert vom Berliner Programm zur Förderung der Chancengleichheit für Frauen in Forschung und Lehre (BCP), wurde die Frage in den Mittelpunkt gestellt, ob sich die Karrierewege und Karriereentscheidungen von Professorinnen und Professoren an Hochschulen für angewandte Wissenschaften unterscheiden und welche Rolle hierbei ein geschlechtergerechtes Organisationsklima spielt.

Der vorliegende Beitrag ist wie folgt gegliedert. Die folgenden Kapitel 3 bis 5 liefern einen theoretischen Überblick über die aktuellen Untersuchungen zum Thema diskriminierungsfreie Hochschule. Organisationskulturelle und -strukturelle Sachverhalte, insbesondere in den MINT-Fächern, werden hierbei näher betrachtet. Kapitel 6 erläutert die Grundlagen der Organisationklimaanalyse. Kapitel 7 schildert die Untersuchung, die die konkrete Situation von Professor*innen an der Beuth Hochschule für Technik analysiert. In Kapitel 8 und 9 werden die Ergebnisse der Studie und die aus den Geschlechtsunterschieden resultierenden Maßnahmen zusammengefasst dargestellt, mögliche Handlungsempfehlungen für die Beuth Hochschule für Technik und technische Hochschulen generell aufgezeigt sowie Ansätze für weiterführende Forschung zu einer gendersensiblen Hochschule diskutiert.

\section{Frauen in der Wissenschaft - Anspruch und Realität}

„Männer und Frauen sind gleichberechtigt. Der Staat fördert die tatsächliche Durchsetzung der Gleichberechtigung von Frauen und Männern und wirkt auf die Beseitigung bestehender Nachteile hin."

(Grundgesetz Artikel 3 Absatz 2)

Die im Grundgesetz verankerte Gleichberechtigung von Mann und Frau garantiert Chancengleichheit der Geschlechter für alle Bereiche des gesellschaftlichen Lebens und schließt somit auch den Bildungsbereich ein. Dieser Anspruch wird pro forma auch eingelöst, sofern es um die Schulbildung und das Studium geht ${ }^{2}$.

Kritischer wird es nach dem Studienabschluss. Die Relation der Geschlechter bleibt zwar bis zur Promotion weitgehend ausgewogen, doch sind nur noch 27 Prozent der Habilitierenden Frauen und ihr Anteil an den vorhandenen Professuren beträgt ganze 16 Prozent, (Braun et al. 2015). Insgesamt liegt der Anteil von Frauen in der Wissenschaft in Deutschland mit 25

\footnotetext{
${ }^{2}$ Laut dem statistischen Bundesamt zeigt ein Blick auf die Geschlechterverteilung der Schulkinder innerhalb des Sekundarbereichs I nach einzelnen Schularten, dass 53,4 Prozent der Mädchen überdurchschnittlich oft Schulen besuchen, die auf einen höheren Schulabschluss hinführen. Dagegen wiesen insbesondere Hauptschulen im Schuljahr 2016/2017 einen überdurchschnittlichen Anteil an Jungen (57 Prozent) auf, ebenso wie die Förderschulen mit 64,8 Prozent Jungen. Die Studienberechtigtenquote der Frauen lag 2016 mit 58 Prozent um rund 12 Prozentpunkte über der der Männer. Diese geschlechtsspezifische Verteilung besteht seit mehr als zehn Jahren.
} 
Prozent unter der weltweiten Quote von 30 Prozent (ebd.). Besonders gering ist der Anteil in den sogenannten MINT-Fächern, also in den mathematisch-naturwissenschaftlich-technisch orientierten Lehr- und Forschungsbereichen mit einem Frauenanteil an Professuren von 15,1 Prozent im Jahr 2018 (s. Abbildung 1). Hier haben sich zwar in den letzten 21 Jahren die Frauenanteile absolut von 4,0 Prozent im Jahr 1997 auf 15,1 Prozent in 2018 verbessert, sind damit aber immer noch extrem niedrig (s. Abbildung 1). Auch im Jahr 2019 kommen in den Ingenieurwissenschaften auf eine Ausschreibung einer Professur durchschnittlich nur 2,5 Bewerbungen von Frauen und 15,2 Bewerbungen von Männern (Sembritzki \& Thiele 2019).

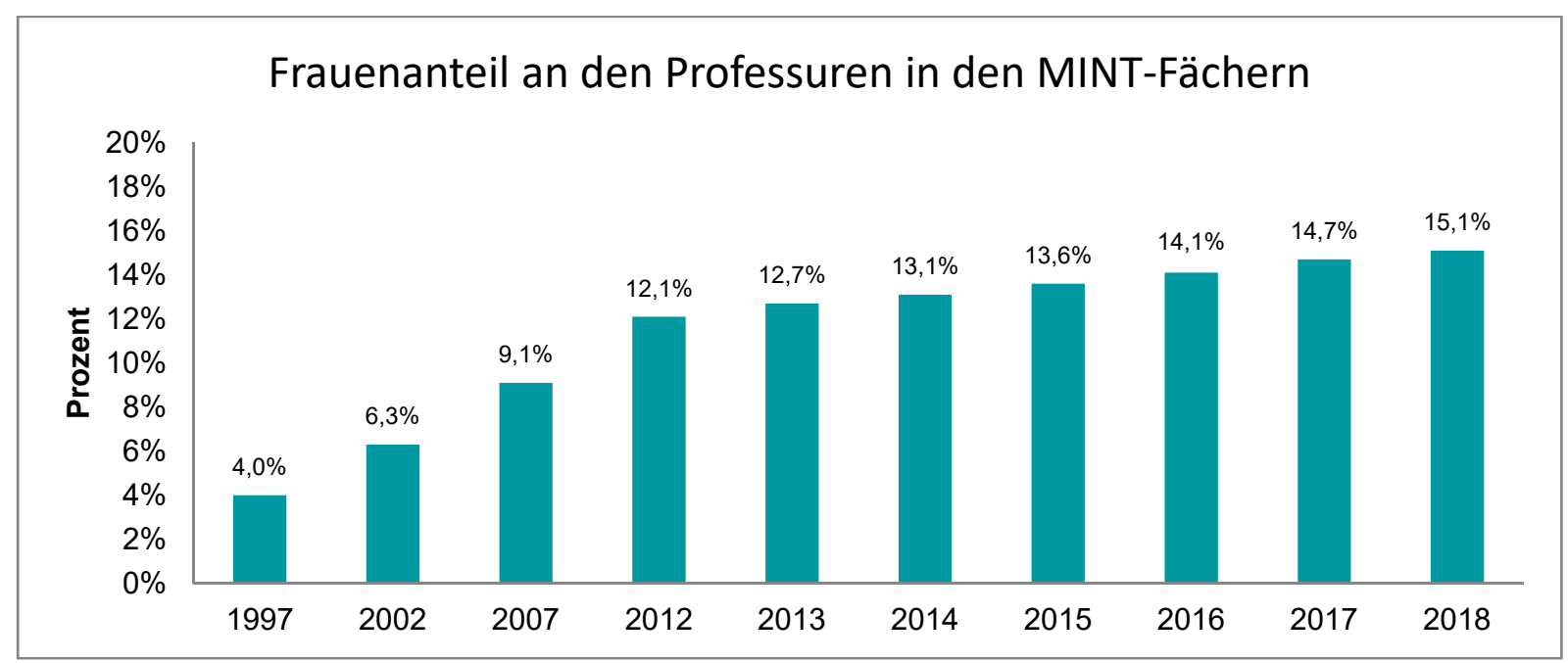

Abbildung 1: Frauenanteil an den Professuren in den MINT-Fächergruppen (Quelle: GWK 2018, S.45; Statistisches Bundesamt 2019, eigene Darstellung)

Abbildung 2 zeigt beispielhaft für die Ingenieurwissenschaften die abnehmenden Frauenanteile über den Karriereverlauf. Die Frauenanteile haben sich zwar innerhalb der Qualifikationsstufen von 1997 bis 2018 geringfügig erhöht, doch bleibt die Abnahme der Frauenanteile mit zunehmender Qualifikationsstufe bestehen. Dieses Phänomen der Geschlechtersegregation, die auch als ,Leaky Pipeline‘ bezeichnet wird, macht deutlich, dass es offensichtlich auch bei vorhandener Einstiegsmotivation zahlreiche Barrieren im Karriereverlauf von Frauen gibt, die zu einer Aufgabe des Berufsziels Professur führen. Die Ursachen der Leaky Pipeline im Wissenschaftsbetrieb sind auf komplexe Wirkmechanismen zurückführen, die sich nach dem Fach, der sozialen Herkunft der Bewerberinnen, der Familiensituation und der individuellen Karriereambitionen unterscheiden können. Allgemein zeigen sich hinsichtlich der soziodemografischen Merkmale, dass Professorinnen eher aus Familien höherer Sozialstatusgruppen kommen (Möller 2015). Im Vergleich zu ihren männlichen Kollegen sind sie häufiger ledig, ihre Ehen sind scheidungsanfälliger, sie haben seltener und weniger Kinder und sie schließen wenn Kinder vorhanden sind - die Familiengründungsphase in einem späteren Lebensalter ab (Schlegel 2006 zitiert nach Sembritzki \& Thiele 2019). 


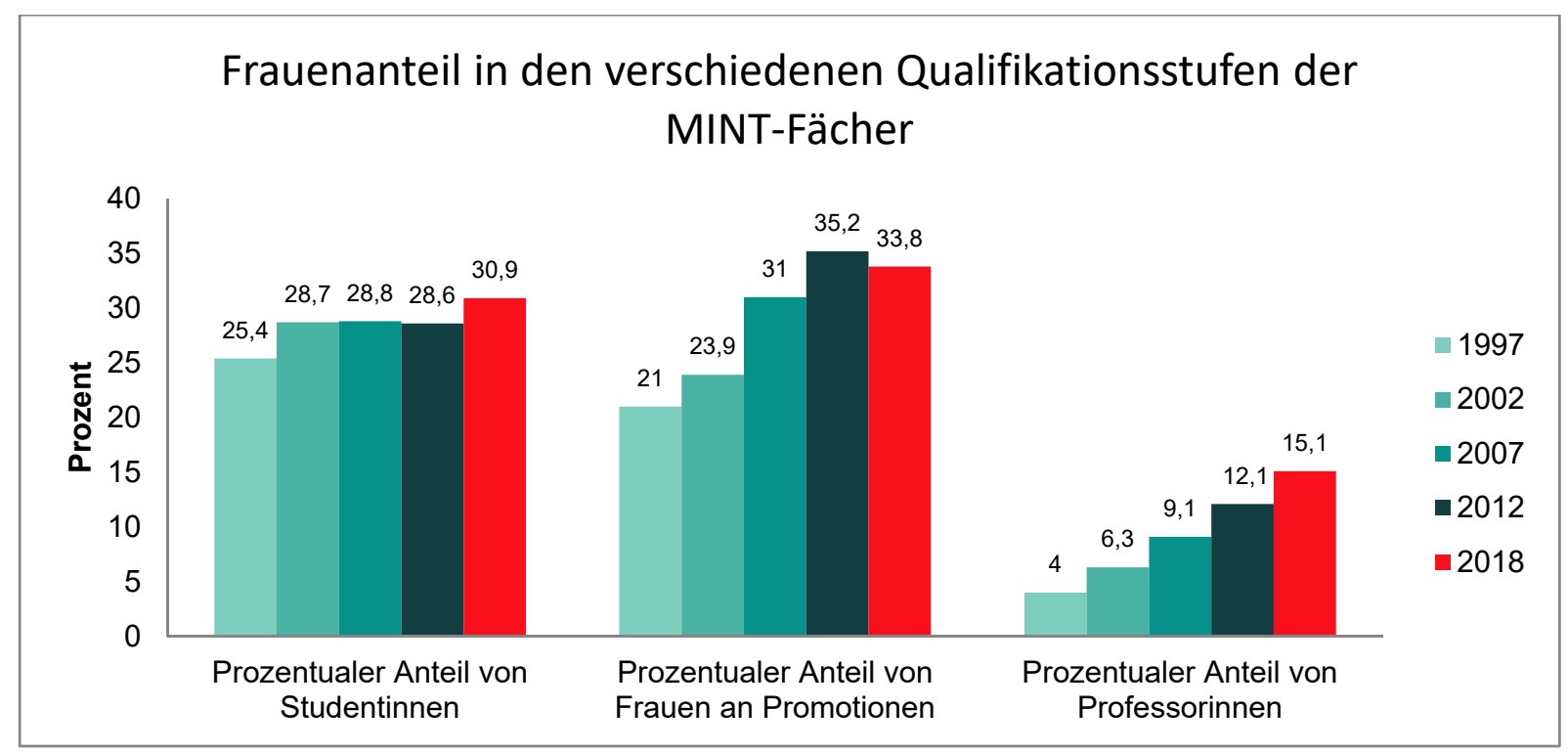

Abbildung 2: Frauenanteile in Prozent in den verschiedenen Qualifikationsstufen der MINT-Fächer (Quelle: GWK 2018, S. 43-45; eigene Darstellung)

\section{Karrieren an Hochschulen für angewandte Wissenschaften}

In Deutschland sind die Hochschulen für angewandte Wissenschaften (folgend HAW) in den Jahren zwischen 1969 und 1971 entstanden und sollten Einrichtungen des tertiären Bildungssektors sein, die Studierende auf wissenschaftlicher Grundlage praxis- und berufsorientiert ausbilden und zu selbständiger Arbeit im Beruf befähigen (Wissenschaftsrat 2002; Bundesministerium für Bildung und Forschung 2004). Der Unterschied zu Universitäten ist der stärkere Anwendungsbezug, eine stärkere Berufsorientierung der Studiengänge, weniger theoretischgrundlagenbezogene Lerninhalte, kürzere Studienzeiten und die unterschiedlichen Zugangsvoraussetzungen ein Studium anzutreten. Die Gründung von HAW basiert unter anderem auf dem Gedanken einer größeren Chancengerechtigkeit, insbesondere für sozial schwache Gruppen mit einer unzureichenden Bildungsförderung (Geißler 2004).

Die Berufung auf eine Professur an den Hochschulen für angewandte Wissenschaften unterscheidet sich von Berufungen an den Universitäten. Eine HAW-Professur setzt eine mindestens dreijährige, meistens fünfjährige Berufserfahrung außerhalb der Hochschule, eine besondere Befähigung zu wissenschaftlicher Arbeit, meist nachzuweisen über eine Promotion sowie Lehrerfahrung voraus (Wissenschaftsrat 2016).

Nach dem Studienabschluss verbleiben Frauen häufiger als Männer im Universitätssystem und arbeiten hier in verschiedenen befristeten Stellen (Metz-Göckel et al. 2016). Sie sind intrasektoral mobiler, wohingegen Männer häufiger in einen außeruniversitären Sektor wechseln (Heusgen 2016). Der längere Verbleib von Frauen im universitären System verschlechtert ihre 
Chancen auf eine HAW-Professur, da sie oftmals nicht die drei Jahre Berufserfahrung außerhalb der Hochschule nachweisen können. Neuere Zahlen aus der Studie von Sembritzki \& Thiele (2019) bestätigen diese Tendenz, nach der die durchschnittliche außeruniversitäre Beschäftigungsdauer ein Jahr kürzer sei, als die der Männer. Sie befragten 443 Neuberufene, davon war ein Drittel weiblich. Unterschiede fänden sich auch bei dem letzten Beschäftigungssektor vor der Bewerbung auf die Professur: 50 Prozent der Männer, aber nur 25 Prozent der Frauen gäben an, aus der Privatwirtschaft zu kommen. Aus der Universität kämen hingegen 14 Prozent der Frauen und nur 10 Prozent der Männer, aus dem öffentlichen Dienst 12 Prozent der Frauen und nur 9 Prozent der Männer (ebd. S.17).

Die Organisationsstrukturen von HAW sind denen der Universitäten ähnlich. Sie sind in ihrer formalen Aufteilung in Fakultäten bzw. Fachbereiche und die hochschulinternen Institute sowie über den akademischen Austausch innerhalb der jeweiligen Fachgebiete auch zwischen Hochschulen miteinander verflochten. Diese Form miteinander vernetzter, teilweise miteinander konkurrierender Interessen und das Streben nach ,Exzellenz' als Garant für das materielle Fortbestehen, kann Konkurrenzsituationen untereinander befördern, was sich nicht zuletzt auf die Stellenbesetzungen auswirkt. So treten - im Sinne der Definition von ,Exzellenz - Kriterien in den Vordergrund, wie die erwiesene Fähigkeit zur Einwerbung von Drittmitteln oder die Publikationshäufigkeit, während andere Qualifikationen, wie etwa die Befähigung zur Lehre und zur Motivation der Studierenden, in den Hintergrund gedrängt werden. Dies erstaunt, da die Lehre in HAW eine hervorgehobene Bedeutung einnimmt, was sich $u$. a. in einem nahezu doppelten Lehrdeputat der HAW-Professuren im Vergleich zu Universitätsprofessuren (18 SWS zu 9 SWS) zeigt.

Das System ,Wissenschaft' schließt Frauen somit nicht per se aus, jedoch richtet sich der Fokus der Aufmerksamkeit eindeutig auf die Personen, die im Kampf um Exzellenz und Anerkennung wichtige Erfolgskriterien erfüllen (Jeschke et al. 2013). Problematisch ist nur, dass bei den Erfolgskriterien, wie sehr gute Vernetzung in die Wirtschaft, hohe Drittmittelquoten und umfangreiche Publikationstätigkeit, Frauen insbesondere in den MINT-Fächern weniger vorzuweisen haben als Männer: Sie verbleiben länger an den Universitäten, gehen seltener in die Wirtschaft, haben häufiger $u$. a. aufgrund nicht linearer Karriereverläufe gerade in den jüngeren Lebensjahren eine geringere Drittmittel- und Publikationsquote und sie verfügen über weniger starke Netzwerke als ihre männlichen Mitbewerber.

Sembritzki \& Thiele (2019) haben in ihrem zweiten Teil ihrer Studie, in der 636 Bewerbungen an HAW ausgewertet wurden, eine durchschnittliche Anzahl von 22 Bewerbungen pro Ausschreibung ermittelt, wovon 22 Prozent von Frauen stammten. Der Frauenanteil an den Bewerbungen ist in den Fächergruppen Ingenieurwissenschaften und Mathematik mit Abstand 
am niedrigsten (hier kommen 2,5 Bewerbungen von Frauen und 15,2 von Männern), wohingegen in den Sprach- und Kulturwissenschaften das Geschlechterverhältnis deutlich ausgeglichener ist (13,3 Bewerbungen von Männern und 10,7 Bewerbungen von Frauen).

Die Tatsache, dass insgesamt verhältnismäßig weniger Bewerbungen von Frauen als Männern auf Professuren erfolgen, wird auch über das unterschiedliche Bewerbungsverhalten von Männern und Frauen erklärt: Frauen bewerben sich selektiver, wohingegen Männer sich mitunter breiter bewerben und sich somit häufiger als Frauen parallel in mehreren Berufungsverfahren befinden (Färber \& Spangenberg 2008, S. 90f.). Damit kommt für Frauen schon der Ausschreibungstext als kritische Größe hinzu: „Je enger man eine Professur ausschreibt, desto mehr verschreckt man die weiblichen Bewerberinnen“" (Sembitzki \& Thiele 2019, S.21). Um den Anteil der Bewerbungen von Frauen zu erhöhen, ist bereits bei der Formulierung der Ausschreibung anzusetzen. Weiter gefasste Ausschreibungsprofile ermuntern Frauen eher sich zu bewerben (Junghans 2012). Es zeigt sich weiterhin, dass sie weniger gut über Berufungsverfahren generell informiert sind und häufiger falsche Vorstellungen über eine HAWProfessur und die geforderten Qualifikationen und die Karriere- und Entwicklungsmöglichkeiten haben (Sembritzki \& Thiele 2019).

Jeschke et al. (2013) konstatieren zusammengefasst, dass Hochschulen auch heute noch bewusst oder unbewusst - Frauen nur unter erschwerten Bedingungen einen Zugang gestatten. Aufgrund schwächerer Netzwerke und der Art ihrer bisherigen Beschäftigungsverhältnisse, werden sie nicht notwendigerweise als relevante, potentiell geeignete Bewerberinnen wahrgenommen (Lind 2006). Da sie selber stärker auf eine Passung der eigenen Qualifikationen und des Stellenprofils achten, bewerben sie sich weniger breit, was die Chancen auf eine Professur im Sinne einer überkritischen Selbstselektion reduziert.

Bisherige Maßnahmen zur Herstellung einer größeren Geschlechtergerechtigkeit innerhalb des Systems Hochschule, wie zum Beispiel über die Einführung von Quotenregelungen oder die Stellensubventionierung über das sogenannten 'Professorinnenprogramm', verfolgen häufig das Ziel einer Erhöhung der Frauenanteile unter Beibehaltung bestehender Strukturen (BMJ 2012; Borgwardt 2013). Eine kreative und konstruktive Wandlung des Systems bewirken sie jedoch nicht, zumal die Einführung von Quotenregelungen gesellschaftlich kontrovers diskutiert werden und sich durchaus auch negativ auf das Image einer Hochschule auswirken kann (Braun et al. 2015). Lösungen liegen deshalb nicht allein in einer letztlich bejahenden Vergrößerung des bereits bestehenden wissenschaftlichen Systems, sondern eher in der Gestaltung von geschlechtergerechten und qualitätsgesicherten Auswahl- und Berufungsverfahren und in der Gestaltung von geschlechtergerechten Organisationsstrukturen und -kulturen 
(Steinweg 2015). Erforderlich hierfür ist eine verstärkte Auseinandersetzung mit Ausschlussmechanismen und mit Genderstereotypen und ihren Folgen, insbesondere bezogen auf die Struktur und die Kultur des Systems ,Wissenschaft' (Reuben et al. 2014).

\section{Wirkmächtige Geschlechterstereotypien}

Auch die Wissenschaft wird getragen und gestaltet von Menschen, die in die jeweiligen Strukturen hineingeboren und in ihrem Sinne sozialisiert wurden. Vorstellungen darüber, was Frauen und Männer ausmacht, existieren in den Köpfen von Akademiker*innen in gleicher Weise wie in denen aller anderen Mitglieder dieser Gesellschaft. Sie bestimmen das Denken und das Handeln, die Definition dessen, wer sie sind und was sie tun, und sie tragen zur Begründung bei, warum Dinge in einer bestimmten Art und Weise getan werden (Banaji \& Greenwald 2013; Isaac et al. 2009). Geschlechterstereotypien bestimmen damit die Organisationskultur eines Systems und liefern auf einer strategischen Ebene den kognitiven wie emotionalen Hintergrund für die operative Seite, die Organisationsstruktur, inklusive aller mit ihr verbundenen und aus ihr resultierenden Entscheidungs- und Handlungsparameter (Braun et al. 2015). Sie wirken nachweislich bei der Auswahl von Personal sowie bei ihrer Eignungsbewertung und sind letztlich auch die Ursache für strukturelle Formen der Diskriminierung. Die Komplexität der Wirkmechanismen wird im Konzept des Gender Bias aufgeschlüsselt.

\subsection{Gender Bias in der Wissenschaft}

„Als Bias werden systematische Verzerrungseffekte bezeichnet, die Wissen und Wahrnehmung beeinträchtigen und benachteiligende Wirkung haben. Die Verzerrungseffekte gehen auf Fehlschlüsse, Vorurteile, Stereotypien, fehlerhafte und vorschnelle Werturteile usw. zurück und prägen individuelle und soziale Wahrnehmungsmuster“ (Jung 2011, S.86). „Als "Gender Bias» wird folglich eine Verzerrung in der Wahrnehmung oder Interpretation von Daten beschrieben, die auf eine stereotype Zuschreibung von Geschlechtsmerkmalen zurückgeht, wobei der Begriff ,Gender‘ die soziale Bedeutung, die den Unterschieden zwischen Frauen und Männern beigemessen wird, hervorhebt" (Liebig und Meyerhofer 2009, S.16).

Der Gender Bias basiert nach Fuchs und Maschewsky-Schneider (2002) auf folgenden Fehlannahmen und Fehlschlüssen: Der Annahme der Unterschiedlichkeit von Frauen und Männer dort, wo keine besteht, der Annahme der Gleichheit von Frauen und Männern dort, wo Gleichheit nicht vorhanden ist und der unterschiedlichen (Be-)Wertung von Faktoren, die bei beiden Geschlechtern auftreten. Diese Fehlannahmen und Fehlschlüsse führen letztlich dazu, dass die spezifische Situation von Frauen nicht, unzureichend oder falsch berücksichtigt wird und liefern die Grundlage von drei Hauptformen des Gender Bias: 
Androzentrismus besteht in der unhinterfragten Adaption einer männlichen Perspektive. Diese kann verschiedene Formen annehmen (Fuchs \& Maschewsky-Schneider 2002, S. 14). Beispielsweise werden Schlüsse für beide Geschlechter auf der Grundlage von rein männlichen Datensätzen gezogen, wie es z. B. bei der Entwicklung von Sicherheitsgurten der Fall war, bei denen zunächst ausschließlich mit männlichen ,Dummies' gearbeitet wurde. In der Versorgungspraxis wurden Ergebnisse klinischer Studien z. B. für medikamentöse Therapien auf Frauen übertragen, obwohl die Wirkungen nur an männlichen Populationen erforscht wurden (Babitsch et al. 2012). Androzentrismus besteht auch, wenn eine an Männern ermittelte oder orientierte Norm aufgestellt wird, an der dann auch Frauen gemessen werden. Beispielsweise sind Typenbezeichnungen erfolgreicher Führungskräfte häufig durch männlich konnotierte Eigenschaftszuschreibungen gekennzeichnet, wie beispielsweise die Attribute durchsetzungsstark, unabhängig oder ehrgeizig.

Von Geschlechtsinsensibilität wird gesprochen, „wenn das biologische oder soziale Geschlecht als wichtiges Anliegen oder Variable ignoriert wird, besonders in Zusammenhängen, in denen Geschlecht bedeutsam ist. Dieses Problem wird auch durch die Begriffe Geschlechterneutralität und Geschlechterblindheit beschrieben“ (ebd. S. 21). Geschlechterinsensibilität besteht beispielsweise, wenn bei der Erforschung von Altersarmut und ihren Determinanten keine geschlechterdifferenzierte Betrachtung erfolgt. So sind bei Frauen Scheidung, Teilzeitarbeit, Erziehungszeiten und Pflegearbeit zentrale Determinanten der Altersarmut (Babitsch, Ducki \& Maschewsky-Schneider 2012).

Doppelte Bewertungsmaßstäbe können offenkundig und verdeckt auftreten. In beiden Fällen aber werden gleichartige oder identische Situationen, Eigenschaften oder Verhaltensweisen für Frauen und Männer jeweils unterschiedlich beurteilt. So wird bezahlte und unbezahlte Arbeit unterschiedlich bewertet, was $u$. a. wesentlich mit der geschlechtlichen Arbeitsteilung verknüpft wird. Auch heute ist es nicht üblich, in der Familienarbeit erworbene Kompetenzen und Fähigkeiten wie z. B. Koordinierungsaufgaben oder pädagogische Aufgaben in Bewerbungssituationen als gleichwertige Vorerfahrungen für ein Stellenprofil anzuerkennen. Wenneras und Wold (1997) haben nachgewiesen, dass Wissenschaftlerinnen um das 2,5-fache produktiver als Wissenschaftler sein mussten, damit sie die gleiche Punktzahl bei der Leistungsbewertung erhielten. Vergleichende Auswertungen von Bewerbungsunterlagen und Gutachten haben zudem gezeigt, dass auch bei identischen Lebensläufen den Männern eher hinreichende Eignung zugeschrieben wird als den Frauen (Steinpreis et al.1999; Schmader et al. 2007; Färber \& Spangenberg 2016). Frauen erhalten eine kritischere Beurteilung durch männliche Vorgesetzte in Empfehlungsschreiben und ihr wissenschaftlicher Eigenbeitrag wird in Gutachten geringer und generell diffuser dargestellt als bei Männern (Braun et al. 2015). 
Die drei Hauptformen verdeutlichen die Vielfalt und Komplexität geschlechtsspezifischer Verzerrungseffekte. Sie können in jeder einzelnen Phase des Wissenschaftsprozesses auftreten und besitzen in Auswahlverfahren und Bewerbungsprozessen eine hohe Relevanz, da hier verschiedene Elemente zusammenwirken. Allgemeine Genderstereotype und -vorurteile können sich also in mehrfacher Hinsicht negativ auf Frauen auswirken: Erstens beeinflussen sie deren Verhalten, zweitens beeinflussen sie das der Männer und Frauen, die Bewertungs- und Entscheidungsmacht besitzen und sorgen damit drittens für Strukturen, die Frauen den Zugang zu akademischen Spitzenpositionen zumindest erschweren.

Geschlecht ist aber nicht die einzige Kategorie, die zu Verzerrungsprozessen in der Leistungsbewertung führen können. Andere soziodemografische und fachspezifische Merkmale treten hinzu. So haben Bornmann et al. (2007) zwar in einer internationalen Meta-Analyse gezeigt, dass im Bereich der Forschungsförderung Männer eine 7 Prozent höhere Bewilligungswahrscheinlichkeit haben als Frauen, allerdings konnten sie selbst diese Ergebnisse in einer erneuten internationalen Meta-Studie nicht replizieren (Marsh et al. 2009). Sie erklären dies darüber, dass die Ergebnisse der vorherigen Studie auf einer Überinterpretation von Gender-Effekten und einer unzureichenden Methodik begründet waren, die u. a. Binnendifferenzierungsmerkmale, wie z. B. Fächergruppen unberücksichtigt ließ. Bestätigung dafür findet sich bei Samjeske (2012). Berücksichtigt wurden hier neben dem Geschlecht z. B. der Status, die institutionelle Herkunft, die Gutachter*innentätikeit und das Fachgebiet. Im Ergebnis findet sie einen Gender Bias in der Bewilligung von Forschungsförderung nur für bestimmte Fächer, Förderjahre oder Institutionen. Die Autorin schließt daraus, dass es unabdingbar ist, bei der Untersuchung wissenschaftlicher Karrieren von Frauen zukünftig das Zusammenwirken verschiedener Faktoren auszuwerten. Geschieht dies, werden z. B. das Geschlecht, die Reputation und der Zugang zu Forschungsressourcen verknüpft, können die Ausschlussmechanismen und die Geschlechterdimensionen in Konzepten, wie der „wissenschaftlichen Exzellenz“ aufgedeckt und präzisere Gegenmaßnahmen entwickelt werden (Beaufays 2012; Kunadt et al. 2014).

\subsection{Technische Fachkulturen - Frau ist ein ,Token', ob sie will oder nicht}

Die Organisations- und Fachkulturen an technischen Hochschulen sind allein durch die Mehrheitsverhältnisse nach wie vor von Männern geprägt. Häufig kommen die Protagonisten selbst aus traditionellen Berufsfeldern der Technik (Molvaer \& Stein 1994; Ihsen 2007), in denen eine männlich dominierte Fachkultur vorherrscht. Wissenschaftlerinnen erfahren innerhalb des Systems entweder geringere Wertschätzung und Bestätigung (Jeschke et al. 2013) oder erleben durch ihre Marginalität eine häufig wohlmeinende „Sonderbehandlung“, die auf die Tatsache zurückzuführen ist, dass Minderheiten in Mehrheitsgruppen immer in besonderem Maße 
auffallen. Nach Kanter (1977) werden Minderheiten (,Tokens') - zum Beispiel Frauen in männerdominierten Organisationen nicht als einzelne Person, sondern als Repräsentant*innen ihrer Gruppe wahrgenommen. Durch die innen zu Teil werdende besondere Aufmerksamkeit sind sie einem besonderen Druck ausgesetzt, überdurchschnittliche Leistungen zu bringen. Scheitern sie, scheitert in den Augen der Mehrheit nicht die Person, sondern die gesamte Gruppe (Zedlacher \& Haas o.J.). Dies gilt generell für Minderheiten in Mehrheitsgruppen, also auch für Männer, die sich in weiblichen Mehrheitsgruppen aufhalten.

Für Frauen in männerdominierten Gruppen bedeutet dies, dass sie immer im besonderen Fokus der Aufmerksamkeit stehen - egal wie bemüht sie sind, nicht aufzufallen. Alles, was sie tun, wird sozusagen zum prototypischen Verhalten ihrer Geschlechtergruppe stilisiert. Diese Prozesse - auch als "doing gender" bezeichnet, bei dem aus der Person mit ihren individuellen Fähigkeiten und Interessen eine Vertreterin ihres Geschlechts wird, die wiederum daran gemessen wird, wie nah oder fern sie dem Geschlechtsbild ihres Gegenübers ist, ist anstrengend, insbesondere dann, wenn Frauen selbst unsicher sind, ob sie da, wo sie sind, richtig sind (Ihsen \& Ducki 2012).

Frauen in männerdominierten Gruppen wählen unterschiedliche Bewältigungsstrategien, um die Differenz zur Mehrheit und ihre Abweichung vom klassischen Rollenbild zu kompensieren. Manche geben dem Rollenzwang nach und übernehmen typisch weibliche - und damit weniger prestigereiche - Aufgaben, die für ihren Karriereweg zwar nicht unbedingt förderlich, aber zumindest wenig konfliktreich sind, da sie nicht gegen traditionelle Rollenbilder verstoßen. Andere wiederum passen sich der Mehrheitsgruppe an, indem sie deren Verhaltensweisen übernehmen und zum Teil sogar übertreiben (Kanter 1977 zitiert nach Zedlacher \& Haas o.J.). Über diese Assimilation oder das Streben danach, können Wissenschaftlerinnen in einen Teufelskreis geraten: Der Mangel an Bestätigung der eignen Arbeit oder die übermäßige Aufmerksamkeit der erbrachten Leistung führt zu einem übererhöhten eigenen Leistungsanspruch (Lind 2006). Dies verzögere die Außendarstellungen z. B. durch Publikationen und reduziere damit die "objektiven Leistungsparameter" (Anzahl der Publikationen). Negative Effekte werden damit in den Erfolgschancen bei Bewerbungen gesehen, die sich wiederum nachteilig auf das Selbstbewusstsein und damit auch auf die Selbstdarstellung auswirken können.

Institutionell bedeutet dies, dass geschlechtliche Monokulturen erhalten bleiben, die die bisher fast unverändert gebliebenen ingenieurwissenschaftlichen Fachkulturen und den männlich geprägten Habitus verfestige und die Gewinnung von Frauen erschwere (Molvaer \& Stein 1994; Ihsen 2007).

Die Möglichkeiten eines Abbaus der asymmetrischen Geschlechterverhältnisse und der Schlüssel zur betrieblichen Gleichstellung lägen - folgt man Kanters Erkenntnissen - in einer 
Erhöhung des Frauenanteils, wobei es, so führt sie aus, für einen Wandel der Geschlechterstereotype mindestens einen Anteil von 15 Prozent Frauen bedürfe und ein Umbruch der Geschlechterpolitik einen Frauenanteil von 30 Prozent voraussetze (Kanter 1977, S. 208 f.). Auch wenn eine Erhöhung der Frauenanteile ein wichtiger Schritt zu mehr Gleichbehandlung ist, reicht er allein nicht aus. Der Gender Bias, wie in Abschnitt 5 aufgezeigt, besteht geschlechtsunabhängig (Moss-Racusin et al. 2012). Das bedeutet, dass eine höhere Anzahl von Frauen in MINT Fächern oder in Auswahl- und Beurteilungsgremien nicht automatisch eine Verbesserung des Systems hinsichtlich gerechterer Verteilung mit sich bringt. In einer Metastudie haben Isaac, Lee und Carnes (2009), die Geschlechtervorurteile in der Bewertung von Bewerbenden in 27 Studien seit 1973 ausgewertet und nachgewiesen, dass Personalentscheiderinnen Bewerberinnen ebenso benachteiligen und bei gleicher Leistung schlechter bewerten, wie Personalentscheider.

Daraus lässt sich schlussfolgern, dass der Weg zu einer geschlechtergerechten Organisation ein langwieriger ist, der sich aus vielen Teilschritten zusammensetzt. Neben der Sichtbarmachung von Token-Mechanismen und ihren Wirkungen bedarf es diskriminierungsfreier Auswahlprozesse und die permanente Arbeit an einem geschlechtergerechten Organisationsklima. Ziel ist schlussendlich die Erhöhung der Frauenanteile vor allem in den Fächern, die einen geringen Frauenanteil aufweisen.

\section{Nutzen und Funktion von Organisationklimaanalysen}

Die Organisationsdiagnose ist ein zielgerichteter, systematischer Prozess, durch den relevante Merkmale und Prozesse der Organisation erfasst werden sollen, die für ihr Funktionieren und ihre Effektivität von Bedeutung sind (Felfe 2019, S. 348). Ziel der Organisationsdiagnose ist es, Handlungsfelder in Organisationen aufzudecken und Maßnahmen der Organisationsentwicklung vorzubereiten, zu begleiten und zu evaluieren (ebd). Eine Organisationsanalyse ist damit Ausgangspunkt und zentraler Bestandteil der Organisationsentwicklung.

Wie schon aufgezeigt, stehen HAW im Zeitalter des Fachkräftemangels in einer besonderen Konkurrenz zur Wirtschaft. Da sie bei den Gehältern, die in der freien Wirtschaft gezahlt werden, nicht mithalten können, sind sie in besonderer Weise gefordert, sich in Hinblick auf andere Merkmale als attraktive Arbeitgeber*in zu positionieren. Solche anderen Merkmale kann die Sinnhaftigkeit der Tätigkeit, familienfreundliche Arbeitsstrukturen und ein angenehmes Organisationsklima sein. Diese Merkmale gilt es in einem systematischen Prozess des Active Sourcing, also der aktiven Ansprache potentiell geeigneter Personen und der Personalgewinnung gezielt zu adressieren. 
Viele HAW verstärken derzeit ihre Anstrengungen im Bereich Personalgewinnung (Rekrutierung) und Mitarbeiterbindung (Commitment, Employer Branding). Um hier entsprechend auf die Bedürfnisse und Besonderheiten der Mitarbeiter*innen reagieren zu können und als Arbeitgeber*in attraktiv zu bleiben, sind deren Sichtweisen und Erwartungen systematisch zu diagnostizieren (Felfe 2019, S. 353f.). Eine besonders relevante Zielgruppe für technische HAW, sind Frauen, die bislang in diesen Bereichen unterrepräsentiert waren. Um auf ihre spezifischen Erwartungen und Bedürfnisse vorbereitet zu sein, sind ihre Sichtweisen systematisch zu diagnostizieren. Dies liefert auch die Grundlage für ein systematisches Diversity Management (ebd).

\section{Organisationsklimaanalyse an der Beuth Hochschule für Technik}

\subsection{Hintergrund und Ziel der Untersuchung}

Die nachfolgend ausgewertete Umfrage zum Organisationsklima an der Beuth Hochschule für Technik wurde über ein Projekt des Berliner ChancengleichheitsProgramms (BCP) initiiert, das als Ziel die Entwicklung von geschlechtergerechten Berufungsstrategien und die Erhöhung des Frauenanteils bei den Professuren hat. Im Projekt „Place to be“ wurden die bisherigen Rahmenbedingungen der Berufungsverfahren als auch die Determinanten eines geschlechtersensiblen Organisationklimas analysiert, um darauf aufbauend die Aspekte in besonderer Weise zu fördern, die die Hochschule als attraktive Arbeitgeber*in für Frauen positioniert. Als Organisationsklima wird hier „die geteilte Wahrnehmung von Mitgliedern einer Organisation von Abläufen und Verfahrensweisen in der Organisation“ verstanden (Kauffeld 2019, S.393). Es hat Auswirkungen darauf, wie zufrieden man mit seiner Arbeit ist, wie stark man sich engagiert und wie stark die Bindung an die Organisation ist (Kauffeld 2019, S.393). Es hat auch Einfluss darauf, wie gerne man die eigene Organisation als Arbeitgeber*in in der eigenen Referenzgruppe weiterempfiehlt.

Im Einzelnen wurden folgende Fragen untersucht:

1. Wie wurden die bisherigen Berufungsverfahren erlebt?

2. Was sind relevante Attraktivitätsdimensionen einer technischen Hochschule?

3. Wie wird das Organisationsklima von denen wahrgenommen, die heute an der Hochschule arbeiten?

Alle Fragen wurden geschlechterdifferenziert ausgewertet.

Die Befragung ist angelehnt an eine Untersuchung zum Organisationsklima im Women In Science And Engineering Leadership Institute' (WISELY) der Universität Wisconsin-Madison, 
wurde aber in einigen Frageformulierungen an die Besonderheiten deutscher HAW angepasst (Sheridan et al. 2007).

Die Studie von Sherdian und ihren Kolleg*innen ist als Langzeitstudie konzipiert, die seit 2003 kontinuierlich wiederholt wird. Ziel ist es hier, Frauen von ihrer Bewerbung an über ihren Eintritt in die Organisation kontinuierlich zum Erleben ihres beruflichen Alltags zu befragen (Sheridan et al. 2007; Devine et al. 2017). Damit sollen u. a. Veränderungen der Organisationskultur dokumentiert, analysiert und mit den Ursprungserwartungen der Befragten abgeglichen werden. Die Untersuchung bezieht sich auch, aber nicht ausschließlich auf Frauen in MINT-Bereichen, wie den Ingenieurwissenschaften.

Anders als in der Studie von Sheridan und ihren Kolleg*innen (2007), die alle Angestellten der Universität befragte, wurden an der Beuth Hochschule ausschließlich Professor*innen befragt. Ferner konnte aufgrund der zu kleinen Fallzahl nur zwischen dem Geschlecht differenziert und keine weiteren soziodemografischen Merkmale berücksichtigt werden. Hinsichtlich der erfassten Attraktivitätsdimensionen unterscheiden sich beide Studien in folgenden Bereichen: In der Studie von Sheridan und ihren Kolleg*innen wurden die Faktoren Einstellungsverfahren, Karriereablauf, berufliche Aktivitäten und die Zufriedenheit mit der Hochschule untersucht. In der vorliegenden Studie wurde neben dem Einstellungsverfahren, den Rekrutierungsstrategien und den aktuellen beruflichen Aktivitäten auch die Führungsintentionen und die Nutzung vorhandener Angebote und Ressourcen sowie die erlebte Work-Life-Balance abgefragt. Ergänzungen von Fragebereichen sind im Wesentlichen den bundesdeutschen Besonderheiten von Berufungsverfahren und Hochschulstrukturen bzw. der Überprüfung der schon vorhandenen Angebote an der Beuth Hochschule geschuldet.

Auf der Grundlage der Ergebnisse der vorliegenden Studie werden Veränderungen und Maßnahmen vorgeschlagen, die die Arbeitgeber*innenattraktivität der Beuth Hochschule erhöhen sollen. Die Wirkung dieser Maßnahmen auf die Beschäftigten soll dann durch Wiederholungsbefragungen evaluiert werden.

\subsection{Inhalt, Aufbau und Durchführung der Untersuchung}

Das Organisationsklima setzt sich aus für die Mitarbeitenden relevanten Merkmalen der Organisation zusammen (Kauffeld 2019): Stimmung unter Kolleg*innen, Beziehung zu Vorgesetzten, Ablauforganisation, Informationen und Mitsprachemöglichkeiten, Zusammenarbeit, Interessenvertretung und betriebliche Leistungen.

In dem vorliegenden Fragebogen gliedern sich die Fragen zu folgenden Themenbereichen:

- zum Einsatz gekommene Rekrutierungsstrategien,

- Einstellungsverfahren,

- Inhalte der beruflichen Aktivitäten und ihre zeitliche Verteilung, 
- Führungsintentionen,

- Wahrgenommene Integration in den Fachbereich,

- Nutzung von vorhandenen Programmen und Ressourcen und

- Work-Life Balance.

Darüber hinaus wurde die Gesamtzufriedenheit erhoben sowie verschiedene soziodemografische Fragen.

Zur tiefergehenden Exploration einzelner Sachverhalte wurden neben standardisierten Antwortformaten auch offene Fragen zu den nachfolgenden Themen gestellt:

Zugang:

In welcher Form wurden Professor*innen direkt zur Bewerbung aufgefordert, falls dies zutrifft? Wie rekrutieren Sie Professorinnen?

Arbeitgeberimage:

Welche Faktoren haben die Entscheidung an die Beuth zu kommen, positiv beeinflusst?

Führung:

Welche Barrieren halten Sie davon ab, Führung zu übernehmen, sofern dies zutrifft?

Work-Life-Balance:

Welche Kinderbetreuungsaspekte halten Sie für generell wichtig?

Unterstützungsangebote:

Welche Unterstützungsangebote der Hochschule für Professor*innen mit hilfs- oder pflegebedürftigen Angehörigen wünschen Sie sich?

Arbeitsklima:

Welche Faktoren tragen zu Ihrer Zufriedenheit bei oder mindern diese?

Kündigungsgründe:

Was sind Ihre Gründe, die Beuth Hochschule verlassen zu wollen?

Sonstiges:

Was möchten Sie noch mitteilen?

Die Befragung richtete sich an alle Professor*innen der Beuth Hochschule für Technik und wurde im Zeitraum vom 29.06.2017 bis zum 24.07.2017 online durchgeführt. Von 304 eingeladenen Professor*innen haben sich 96 an der Umfrage beteiligt. Das entspricht einem Rücklauf von 31,6 Prozent. Darunter waren 21 Professorinnen und 67 Professoren. Die OnlineUmfrage wurde mit der Evaluationssoftware EvaSys durchgeführt und mithilfe der Statistiksoftware SPSS und MAXQDA quantitativ und qualitativ ausgewertet. Die Unterschiede zwischen Professorinnen und Professoren, hinsichtlich ihrer Bewertung einzelner Sachverhalte, wurden mithilfe des Mann-Whitney-U-Tests ${ }^{3}$ auf Signifikanz ${ }^{4}$ geprüft.

Betrachtet man die vorliegende Stichprobe im Vergleich zur Grundgesamtheit der Professorenschaft der Beuth Hochschule wird deutlich, dass die Antwortenden die Grundgesamtheit 
nur teilweise repräsentieren. Innerhalb der Stichprobe sind die Professorinnen leicht überrepräsentiert. Über die nachstehende Tabelle 1 ist ersichtlich, dass die Antwortenden mit 10,9 Jahren im Durchschnitt etwas länger an der Hochschule als Professor*in beschäftigt sind, als die gesamte Professorenschaft der Hochschule (8,0 Jahre). Für eine Betrachtung einzelner Fachbereiche ist der Rücklauf nicht ausreichend groß. Ungefähr ein Drittel der Antwortenden hat zur Dauer der Beschäftigung keine Angabe gemacht. Die Größe und die Zusammensetzung der Stichprobe werden bei der Auswertung und Interpretation der Ergebnisse berücksichtigt.

Tabelle 1: Grundgesamtheit versus Stichprobe ${ }^{5}$

\begin{tabular}{|l|l|l|}
\hline \multicolumn{1}{|c|}{ Merkmal } & \multicolumn{1}{|c|}{ Grundgesamtheit } & \multicolumn{1}{c|}{ Stichprobe $^{6}$} \\
\hline w (weiblich) & $20,6 \%$ & $25 \%(n=88)$ \\
\hline durchschnittliche Beschäftigungsdauer & 8,0 Jahre & 10,9 Jahre $(n=87)$ \\
\hline Kind/er & $70,2 \%$ & $64,0 \%(n=89)$ \\
\hline
\end{tabular}

\subsection{Ergebnisse}

\subsubsection{Einstellungsverfahren}

In der Absicht zu analysieren, ob es funktionierende wissenschaftliche Netzwerke gab, die zur Akquise von potentiellen Kandidat*innen genutzt werden können, wurden die Professor*innen danach gefragt, ob sie direkt angesprochen und zur Bewerbung aufgefordert wurden. Insgesamt gaben ungefähr ein Viertel (24 Prozent) der Bewerbenden an, auf eine direkte Ansprache hin eingeladen worden zu sein. Hierbei zeigten sich geringfügige Unterschiede zwischen Frauen und Männern: 23,8 Prozent der Professorinnen und 28,4 Prozent der Professoren gaben an, dass sie direkt zur Bewerbung aufgefordert wurden. Die Netzwerke der Professoren schienen etwas intensiver zur Anwerbung genutzt worden zu sein, als die der Frauen.

\footnotetext{
${ }^{5}$ Quelle: Statistik des Personalreferats, Ergebnisse der Umfrage

${ }^{6}$ Die im Text genannten Prozent- oder Durchschnittsangaben, beziehen sich immer auf die Fälle, für die ein gültiger Wert vorliegt (n).
} 


\section{Direkte Aufforderung zur Bewerbung}

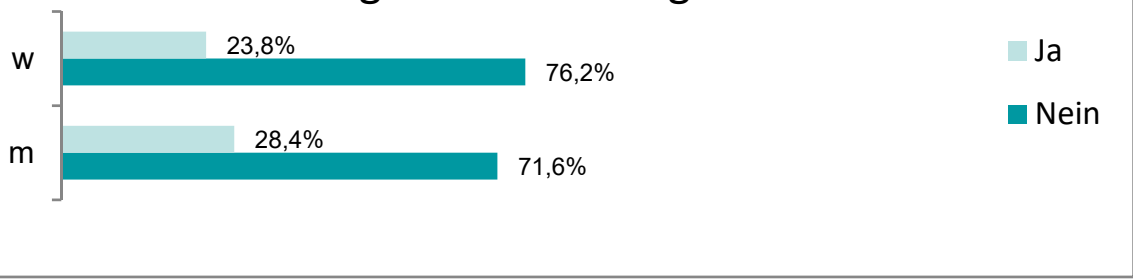

Legende: Angaben in Prozent, getrennt nach männlich $(m, n=75)$ und weiblich $(w, n=21)$

Abbildung 3: Direkte Aufforderung zur Bewerbung an der Beuth Hochschule

Die anschließenden Fragen zur Zufriedenheit mit verschiedenen Aspekten des Einstellungsprozesses dienten dazu, Verbesserungspotentiale für zukünftige Berufungsprozesse zu identifizieren.

\section{Zufriedenheit mit dem Berufungsverfahren}

Meine Kontakte mit der Berufungskommission waren positiv $(n=94)$

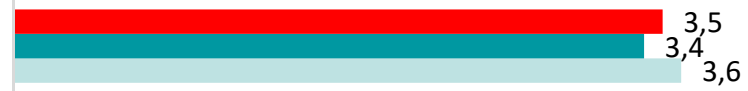

Die Berufungskommission tat ihr Bestes, mir Ressourcen (z.B. Wissen zur HS, techn. Equipment für den Vortrag, etc.) zur Verfügung zu stellen $(n=94)$

Ich war mit dem Einstellungsprozess insgesamt zufrieden $(n=95)$

Mir war der Prozessablauf im Vorhinein bekannt ( $n=93$ )

Ich konnte vorab über den Berufungsprozess Informationen einholen von einem Hochschulmitglied $(n=80)$

Die online zur Verfügung gestellten Informationen für potentielle Bewerbende fand ich informativ $(n=59)$

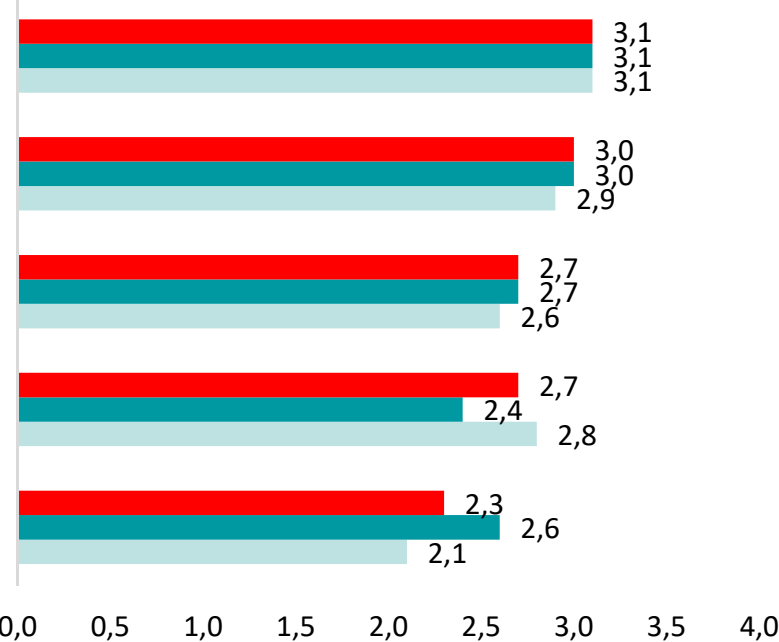

durchschnittliche Zustimmung gesamt durchschnittliche Zustimmung w durchschnittliche Zustimmung $\mathrm{m}$

Legende: n: Anzahl Antworten gesamt; Skala von 0 („stimme nicht zu“) bis 4 („stimme sehr zu“); w=weiblich, $m=$ männlich (Quelle: EvaSys Ergebnisbericht, eigene Darstellung)

Abbildung 4: Befragungsergebnisse zum Bewerbungsprozess

Wie die Abbildung 4 zeigt, lag das größte Verbesserungspotential im technischen Bereich und nicht im Bereich des persönlichen Kontakts. Während der persönliche Kontakt und das Bemühen der einzelnen Hochschulmitglieder durchaus positiv gesehen wurden, war der Informationsfluss optimierbar. Sowohl online, als auch im persönlichen Kontakt bewerteten die Antwortenden ihren Bewerbungsprozess als nicht informationsreich und transparent. In dieser Beurteilung gab es keine signifikanten Geschlechtsunterschiede. 


\subsubsection{Entscheidungsfaktoren, die zur Bewerbung geführt haben}

Im darauffolgenden Abschnitt wurden die Professor*innen nach den fünf wichtigsten Entscheidungsfaktoren gefragt, um eine Professur an der Beuth Hochschule anzunehmen.

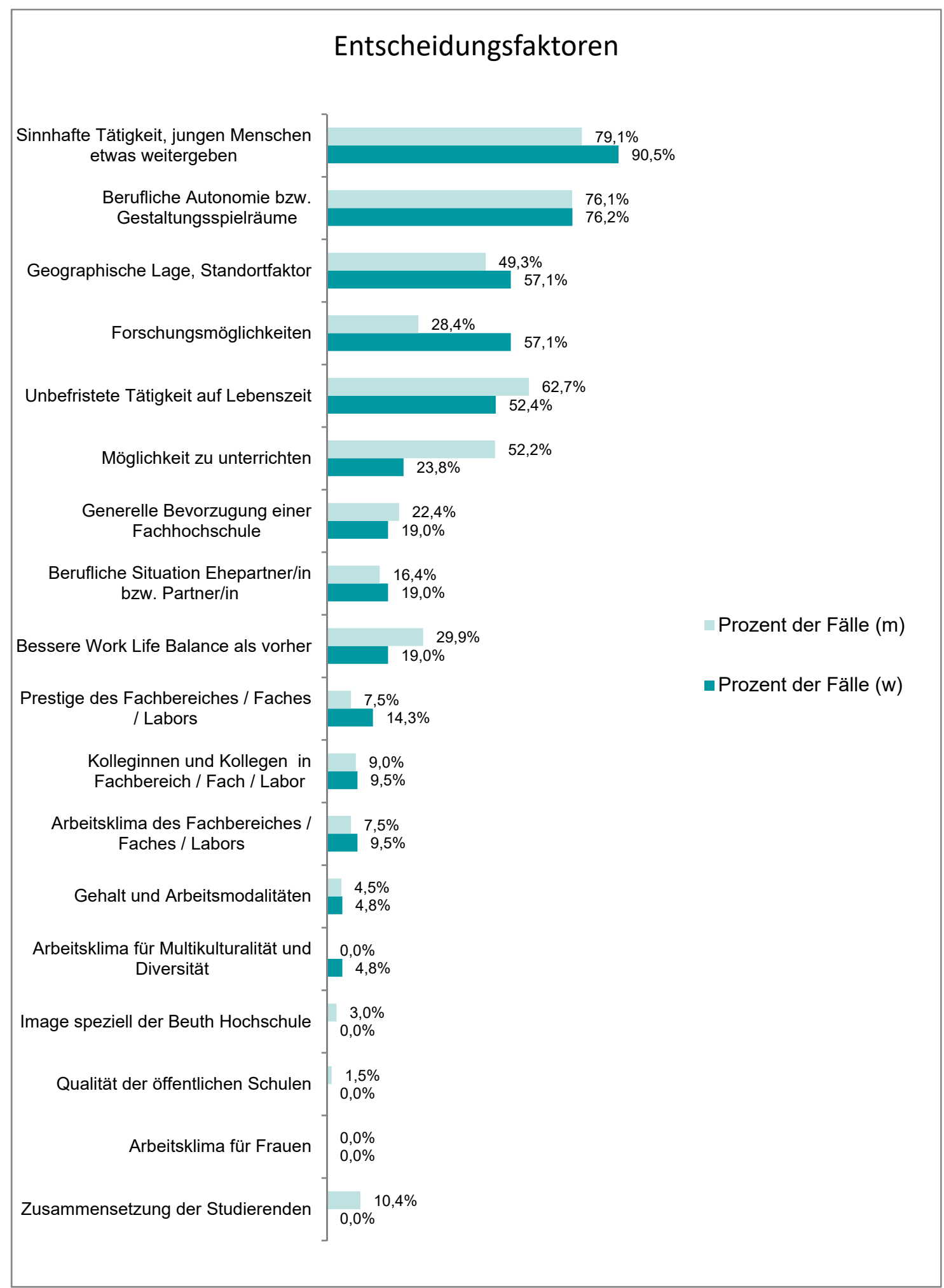

Legende: Angaben in Prozent, getrennt nach männlich (m, $n=75)$ und weiblich (w, n=21) (Quelle: EvaSys Ergebnisbericht, eigene Darstellung)

Abbildung 5: Entscheidungsfaktoren 
Generell waren sich Männer und Frauen bis auf wenige Ausnahmen einig. Mehr als drei Viertel aller Befragten nannten vor allem die Autonomie und die sinnstiftenden Aspekte ihrer Tätigkeit, wie ihr Wissen an junge Menschen weiter zu geben als relevanten Entscheidungsfaktor. Aber auch die günstige geographische Lage der Hochschule und eine unbefristete Tätigkeit auf Lebenszeit war beiden Geschlechtern wichtig, wobei sie für Professoren deutlich wichtiger war als für Professorinnen.

Der größte Unterschied zwischen den Geschlechtern lag interessanterweise bei den Forschungsmöglichkeiten. Prozentual gesehen gaben fast doppelt so viele Professorinnen $(57,1$ Prozent der Frauen aber nur 28,4 Prozent der Männer) an, dass die Möglichkeit zu forschen für sie einen wichtigen Entscheidungsgrund für eine Professur dargestellt hat (Abbildung 5 ). ${ }^{7}$

Ein deutliches Ergebnis zeigte sich in Hinblick auf das Image der Beuth Hochschule für Technik Berlin. Es spielte bei der Entscheidung sich zu bewerben keine wesentliche Rolle, wie auch das Arbeitsklima für Multikulturalität und Diversität für beide Geschlechter irrelevant für die Wahl der Beuth Hochschule für Technik als Arbeitgeber*in war.

Die hier gefundenen Ergebnisse zu den Entscheidungsmotiven befinden sich in Übereinstimmung mit den Ergebnissen der deutschlandweiten Studie von Sembritzki und Thiele (2019), in der eine große Selbstbestimmtheit der Arbeit, die zeitliche Flexibilität und die Arbeit mit jungen Menschen als attraktive Merkmale einer HAW-Professur gesehen wurden. Ferner wurden in der Studie von Sembritzki und Thiele auch die Zusammenarbeit in regionalen Netzwerken und die Kooperation mit kleinen und mittleren Unternehmen als attraktive Merkmale angesehen.

\subsubsection{Berufliche Tätigkeiten (Verteilung der Arbeitszeit, verfügbare Ressourcen)}

Der nächste Punkt soll Aufschluss darüber geben, wie sich die Arbeitszeit der Professor*innen in der Realität auf Lehre, Forschung, Beratung und akademische Selbstverwaltung verteilt hatte.

\footnotetext{
${ }^{7}$ Die Befragungsergebnisse wurden als Anlass genommen, das Geschlechterverhältnis der tatsächlich forschungsaktiven Professor*innen zu überprüfen. An der Beuth Hochschule gab es nach Angaben des Forschungsreferats der Beuth Hochschule 2017105 aktive Forscher*innen, davon waren 29 Frauen und 76 Männer. Bezogen auf die absolute Anzahl der Professoren und Professorinnen (230 männlich und 60 weiblich) sind also 48,3 Prozent der Professorinnen und 29,2 Prozent der Professoren forschungsaktiv. Das bedeutet, dass Frauen tatsächlich deutlich häufiger forschungsaktiv sind als ihre männlichen Kollegen.
} 
Tabelle 2: Verteilung der Arbeitszeit

(Angaben in Prozent, getrennt nach männlich (m) und weiblich (w))

\begin{tabular}{|c|c|c|c|c|}
\hline \multirow{2}{*}{ Berufliche Tätigkeiten } & \multicolumn{2}{|c|}{$\begin{array}{c}\text { tatsächlich verbrachte } \\
\text { Arbeitszeit }\end{array}$} & \multicolumn{2}{c|}{ gewünschte Arbeitszeit } \\
\cline { 2 - 5 } & $\mathrm{m}$ & $\mathrm{w}$ & $\mathrm{m}$ & $\mathrm{W}$ \\
\hline Lehre & 64,8 & 57,6 & 56,5 & 51,0 \\
\hline $\begin{array}{c}\text { Forschung } \\
\text { (inklusive Veröffentlichungen) }\end{array}$ & 9,8 & 14,7 & 22,4 & 26,3 \\
\hline $\begin{array}{c}\text { Beratung von Studierenden, } \\
\text { Mentoring }\end{array}$ & 11,9 & 14,3 & 12,5 & 14,5 \\
\hline Akademische Selbstverwaltung & 14,2 & 15,3 & 9,2 & 10,0 \\
\hline
\end{tabular}

Bei der Verteilung der Arbeitszeit zeigten sich Geschlechtsunterschiede: Die befragten Professorinnen gaben an, dass sie weniger Arbeitszeit mit Lehre verbracht haben und mehr mit Forschung, Beratung und akademischer Selbstverwaltung als ihre männlichen Kollegen. Dieses Ergebnis passt zu dem vorherigen Befragungsergebnis, nach dem Frauen die Möglichkeit zu forschen wichtiger ist als den Männern. Sowohl die Professorinnen als auch die Professoren gaben an, dass sie weniger Zeit mit Lehre verbringen möchten und mehr mit Forschung. Im Folgenden wurde die generelle Zufriedenheit der Professor*innen an der Beuth Hochschule für Technik in Beziehung zu der tatsächlichen und der gewünschten Arbeitszeit gesetzt (siehe Tabelle 3 und 4).

Wird die Differenz zwischen den Werten der tatsächlichen und der gewünschten Arbeitszeit in den verschiedenen Tätigkeitsfeldern betrachtet, ergeben sich keine großen Diskrepanzen zwischen Frauen und Männern (Tabelle 5). In Bezug auf die Zeit, die auf Lehre verbracht wird, wünschen sich beide Geschlechter eine geringfügige Absenkung ihrer tatsächlichen Arbeitszeit um etwa 10 Prozent. Für Forschung hätten hingegen Professorinnen und Professoren gerne nahezu doppelt so viel Zeit, als sie real investieren können. Die aufgebrachte Arbeitszeit für Beratung von Studierenden und Mentoring entspricht in etwa den „Wunschzeiten“, während die tatsächlich aufgebrachte Zeit in der akademischen Selbstverwaltung bei Professorinnen und Professoren gerne um ein Drittel reduziert werden könnte.

Betrachtet man abschließend das gesamte Zeitbudget einer Person, verteilt über die verschiedenen Arbeitsfelder, ergibt sich für Professorinnen und Professoren eine einheitliche Präferenz: Professorinnen und Professoren wünschen sich etwas weniger Zeitinvestitionen in die Lehre und die akademische Selbstverwaltung zugunsten der Forschung. 
Tabelle 3: Zusammenhang zwischen Zufriedenheit und Verteilung der tatsächlichen Arbeitszeit (Angaben in Prozent, getrennt nach männlich $(\mathrm{m})$ und weiblich $(\mathrm{w})$ )

\begin{tabular}{|c|c|c|c|c|c|}
\hline Zufriedenheit & Geschlecht & Lehre & Forschung & Beratung & $\begin{array}{c}\text { Akademische } \\
\text { Selbstverwal- } \\
\text { tung }\end{array}$ \\
\hline \multirow{2}{*}{ sehr zufrieden } & $\mathrm{m}$ & 73,8 & 8,6 & 8,9 & 10,6 \\
\cline { 2 - 6 } & $\mathrm{w}$ & 58,0 & 12,0 & 10,0 & 20,0 \\
\hline \multirow{2}{*}{ zufrieden } & $\mathrm{m}$ & 62,5 & 10,7 & 12,4 & 15,1 \\
\cline { 2 - 6 } & $\mathrm{w}$ & 60,4 & 15,5 & 15,8 & 11,4 \\
\hline \multirow{2}{*}{$\begin{array}{c}\text { weniger } \\
\text { zufrieden }\end{array}$} & $\mathrm{m}$ & 64,4 & 8,6 & 14,4 & 13,8 \\
\cline { 2 - 6 } & $\mathrm{w}$ & 48,8 & 16,3 & 15,0 & 20,0 \\
\cline { 2 - 6 } unzufrieden & $\mathrm{m}$ & 66,8 & 8,6 & 10,4 & 13,0 \\
\cline { 2 - 6 } & $\mathrm{w}$ &. &. &. &. \\
\hline
\end{tabular}

Setzt man die Zufriedenheit der Professor*innen mit der Verteilung der tatsächlichen Arbeitszeit in Beziehung, zeigten sich folgende Unterschiede. Diejenigen Professoren, die angegeben haben „sehr zufrieden“ an der Beuth Hochschule für Technik zu sein, verbrachten die meiste Zeit mit Lehre (73,8 Prozent) (Tabelle 3). Frauen gaben im Gegensatz zu Männern nie an, unzufrieden zu sein. Sie sind generell höchstens „weniger zufrieden“. Professorinnen, die viel Zeit mit Forschung verbringen wollen (30,0 Prozent), gaben häufiger an, dass Sie „weniger zufrieden" sind (siehe Tabelle 3).

Tabelle 4: Zusammenhang zwischen Zufriedenheit und Verteilung der gewünschten Arbeitszeit (Angaben in Prozent, getrennt nach männlich $(\mathrm{m})$ und weiblich $(\mathrm{w})$ )

\begin{tabular}{|c|c|c|c|c|c|}
\hline Zufriedenheit & Geschlecht & Lehre & Forschung & Beratung & $\begin{array}{c}\text { Akademische } \\
\text { Selbstverwal- } \\
\text { tung }\end{array}$ \\
\hline \multirow{2}{*}{ sehr zufrieden } & $\mathrm{m}$ & 65,9 & 16,9 & 10,1 & 9,9 \\
\cline { 2 - 6 } & $\mathrm{w}$ & 49,0 & 23,0 & 12,0 & 16,0 \\
\hline \multirow{2}{*}{ zufrieden } & $\mathrm{m}$ & 53,8 & 23,5 & 12,6 & 10,0 \\
\cline { 2 - 6 } & $\mathrm{w}$ & 52,5 & 26,4 & 16,3 & 7,7 \\
\hline \multirow{2}{*}{ weniger zufrieden } & $\mathrm{m}$ & 60,0 & 20,0 & 12,5 & 7,5 \\
\cline { 2 - 6 } & $\mathrm{w}$ & 48,8 & 30,0 & 12,5 & 8,8 \\
\hline \multirow{2}{*}{ unzufrieden } & $\mathrm{m}$ & 56,0 & 24,0 & 15,0 & 6,6 \\
\cline { 2 - 6 } & $\mathrm{w}$ & &. &. &. \\
\hline
\end{tabular}

männlich $(m) n=66$, weiblich $(w) n=21$ *Keine der befragten Professorinnen gab an, unzufrieden zu sein 
Tabelle 5: Differenzwerte zwischen tatsächlicher und gewünschter Arbeitszeit von zufriedenen und weniger zufriedenen Professor*Innen*

(Angaben in Prozent, getrennt nach männlich $(\mathrm{m})$ und weiblich $(\mathrm{w})$ )

\begin{tabular}{|c|c|c|c|c|c|}
\hline Zufriedenheit & Geschlecht & Lehre & Forschung & Beratung & $\begin{array}{c}\text { Akademische } \\
\text { Selbstverwal- } \\
\text { tung }\end{array}$ \\
\hline \multirow{2}{*}{ Differenz zufrieden } & $\mathrm{m}$ & 12,25 & 14,7 & 1,3 & 3,25 \\
\cline { 2 - 6 } & $\mathrm{w}$ & 12,95 & 16,45 & 2,25 & 5,85 \\
\hline \multirow{2}{*}{ Differenz unzufrieden } & $\mathrm{m}$ & 9,8 & 19,1 & 0,4 & 9,5 \\
\cline { 2 - 6 } & $\mathrm{w}$ & 0,0 & 13,7 & 2,5 & 11,2 \\
\hline
\end{tabular}

Männlich $(m) n=66$, weiblich $(w) n=21{ }^{*}$ Für die Tabelle wurden Mittelwerte von sehr zufrieden und zufrieden als auch von weniger zufrieden und unzufrieden gebildet.

\section{Verfügbare Ressourcen}

Generell waren die Professorinnen zufriedener oder gleich zufrieden in der Beurteilung ihrer verfügbaren Ressourcen, nur mit den zugeteilten Laborflächen waren sie weniger zufrieden als ihre männlichen Kollegen. Auffällig war, dass Professor*innen sich durch ihre Kolleg*innen sehr gut unterstützt fühlten, was auf ein gutes Arbeitsklima an der Hochschule hingedeutet hat. Wohingegen die Verfügbarkeit von Reisemitteln eher negativ gesehen wurde.

\section{Einschätzung der Ressourcenausstattung}

Ich habe ausreichend Reisemittel $(n=73)$

Ich habe Kollegen/innen in der Hochschule, die ähnliche Forschung durchführen ( $n=72)$

Ich erhalte regelmäßige Instandhaltungen und Upgrades der technischen Ausstattung $(n=85)$

Ich habe ausreichend administrative Unterstützung (z.B.

Fachbereichsverwaltungen, Personalreferat) $(n=87)$

Ich habe das Equipment, das ich benötige, um meine

Forschung und Lehre adäquat zu leiten. $(n=87)$

Ich habe genügend Laborfläche zur Verfügung $(n=69)$

Ich erhalte die technische Unterstützung, die ich benötige $(n=86)$
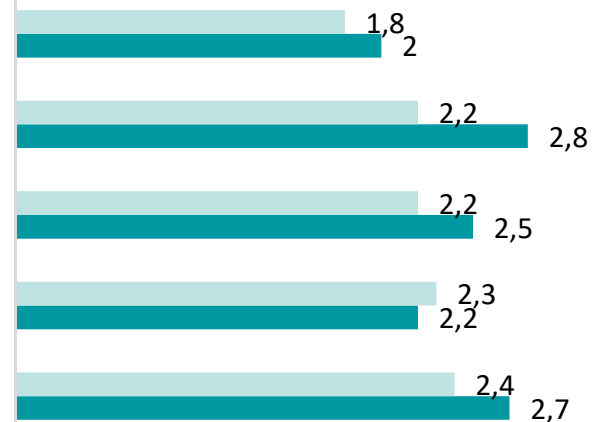

(1)

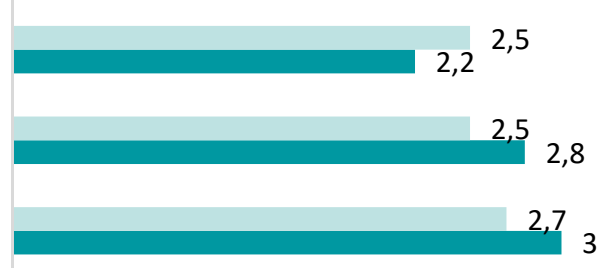

Ich habe Kollegen/innen, die mir Unterstützung anbieten, wenn ich es brauche. $(n=87)$

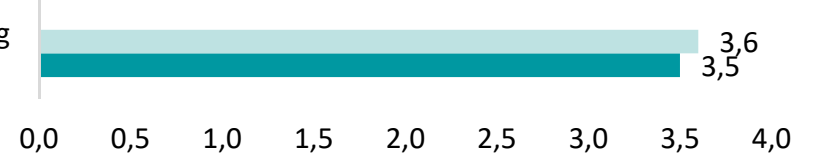

Durchschnittliche Zustimmung Männer

Durchschnittliche Zustimmung Frauen

Legende: Zustimmung von 0 („stimme nicht zu“) bis 4 („stimme sehr zu“); n: Anzahl Antworten gesamt (Quelle: EvaSys Ergebnisbericht, eigene Darstellung)

Abbildung 6: Meinungsbild zu den verfügbaren Ressourcen 
In Bezug auf die Mitarbeit der Professor*innen in der akademischen Selbstverwaltung fiel auf, dass Frauen häufiger in der akademischen Selbstverwaltung mitgearbeitet haben, als ihre männlichen Kollegen. Ein Grund hierfür kann die Quotierung in den Gremien sein, neben einem positiven Stichprobeneffekt. Auch für die Zukunft kann sich ein größerer Anteil der Professorinnen vorstellen, in der akademischen Selbstverwaltung mitzuarbeiten, als das für die Professoren der Fall ist (85,7 Prozent zu 68,2 Prozent). 31,8 Prozent der antwortenden Professoren möchte in der Zukunft nicht in diesem Bereich aktiv sein. Die mangelnde Bereitschaft in der akademischen Selbstverwaltung tätig zu werden, erschwert möglicherweise die Besetzung von Gremien.

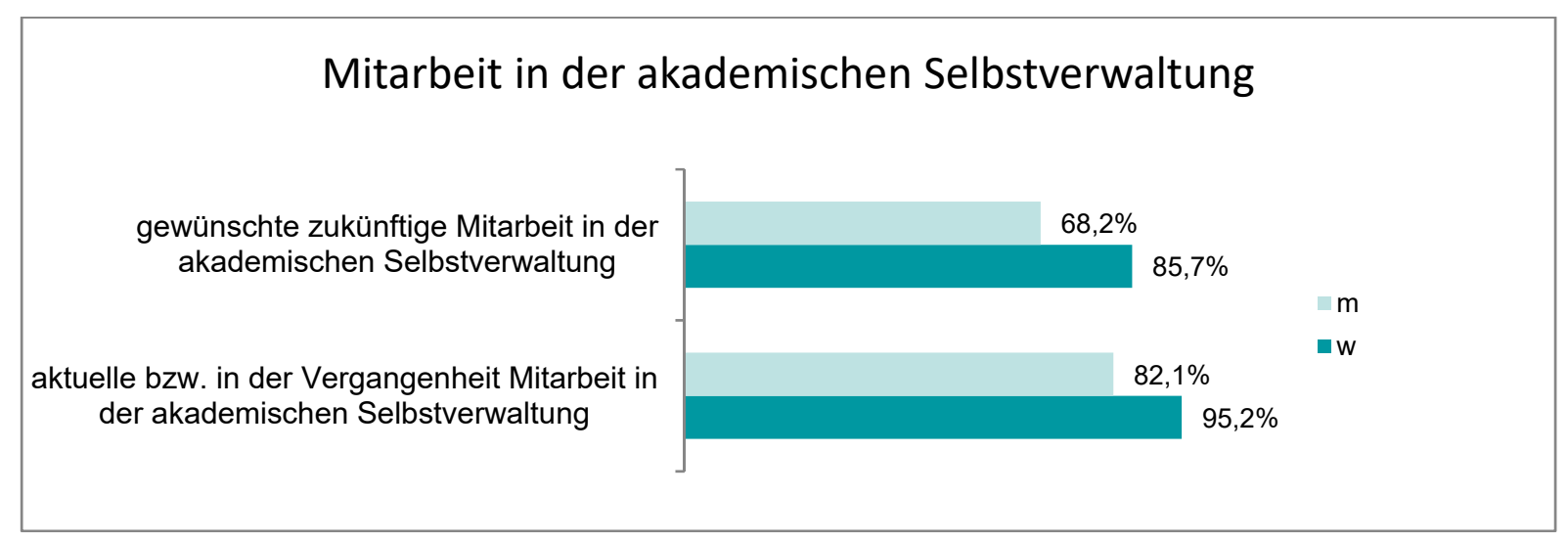

Legende: Angaben in Prozent, getrennt nach männlich $(m, n=75)$ und weiblich $(w, n=21)$

Abbildung 7: Mitarbeit in der akademischen Selbstverwaltung

Insgesamt wird in dem Bereich „berufliche Tätigkeiten“ ersichtlich, dass die Abweichungen der realen Arbeitszeitverteilungen in Bezug auf Lehre, Forschung und Beratung und akademische Selbstverwaltung und Wunschverteilungen bei beiden Geschlechtern eindeutig darauf hinweisen, Veränderungen vorzunehmen. Beide Geschlechter möchten den Zeitanteil für Forschung auf Kosten der Lehre deutlich erhöhen und sehen Optimierungsbedarfe bei der Verfügbarkeit von technischen, räumlichen und personellen Ressourcen.

\subsubsection{Integration in den Fachbereich}

Generell fühlten sich Professor*innen an der Beuth Hochschule für Technik von ihren Kolleg*innen wertgeschätzt. Professorinnen stimmten der Aussage „Kolleginnen und Kollegen holen meine Meinung bei Arbeitsthemen ein“ jedoch eher zu, als ihre männlichen Kollegen. Die Professorinnen fühlten sich zwar stärker wertgeschätzt in ihrer Arbeit, aber eben auch stärker belastet mit Aufgaben, die nicht zu ihrem Rollenverständnis als Professorin passten. Die Unterschiede zwischen den Professor*innen waren jedoch nicht signifikant und damit möglicherweise zufällig. Die Aussage „Ich fühle mich in informelle Netzwerke in meinem Fachbereich integriert" bewerteten die Befragten auf einer Skala von $1=$ "stimme nicht zu" bis $4=$ "stimme sehr zu" beispielsweise durchschnittlich ,nur" mit einem Wert von 2,8. 


\section{Integration im Fachbereich}

Ich muss viele Aufgaben machen, die nicht zu meinem Aufgabenverständnis gehören. $(n=86)$

Ich treffe auf ungeschriebene Gesetze darüber, wie man in meinem Fachbereich interagieren sollte. $(n=76)$

Ich mache viele Arbeiten, die in meinem Fachbereich nicht formal anerkannt werden. $(n=84)$

Ich fühle mich in informelle Netzwerk in meinem Fachbereich integriert. $(n=85)$

Ich fühle mich generell an der Beuth Hochschule integriert. $(n=86)$

Ich fühle mich in meinem Fachbereich integriert. $(n=87)$

Kollegen/innen holen meine Meinung zu Arbeitsthemen ein (Lehre, Forschung, Administration). ( $n=86$ )

Ich habe das Gefühl, ich "passe" in meinen Fachbereich. $(n=83)$

Ich habe das Gefühl, dass meine Kollegen/innen meine Arbeit wertschätzen. $(n=83)$

Ich werde von meiner/m Dekan/in und Prodekan/in mit Respekt behandelt. $(n=84)$

Ich werde von meinen Studierenden mit Respekt behandelt. $(n=88)$

Ich werde von meinen Kollegen/innen mit Respekt behandelt. $(n=87)$

Ich werde von Mitarbeitenden des Fachbereichs mit Respekt behandelt. $(n=87)$

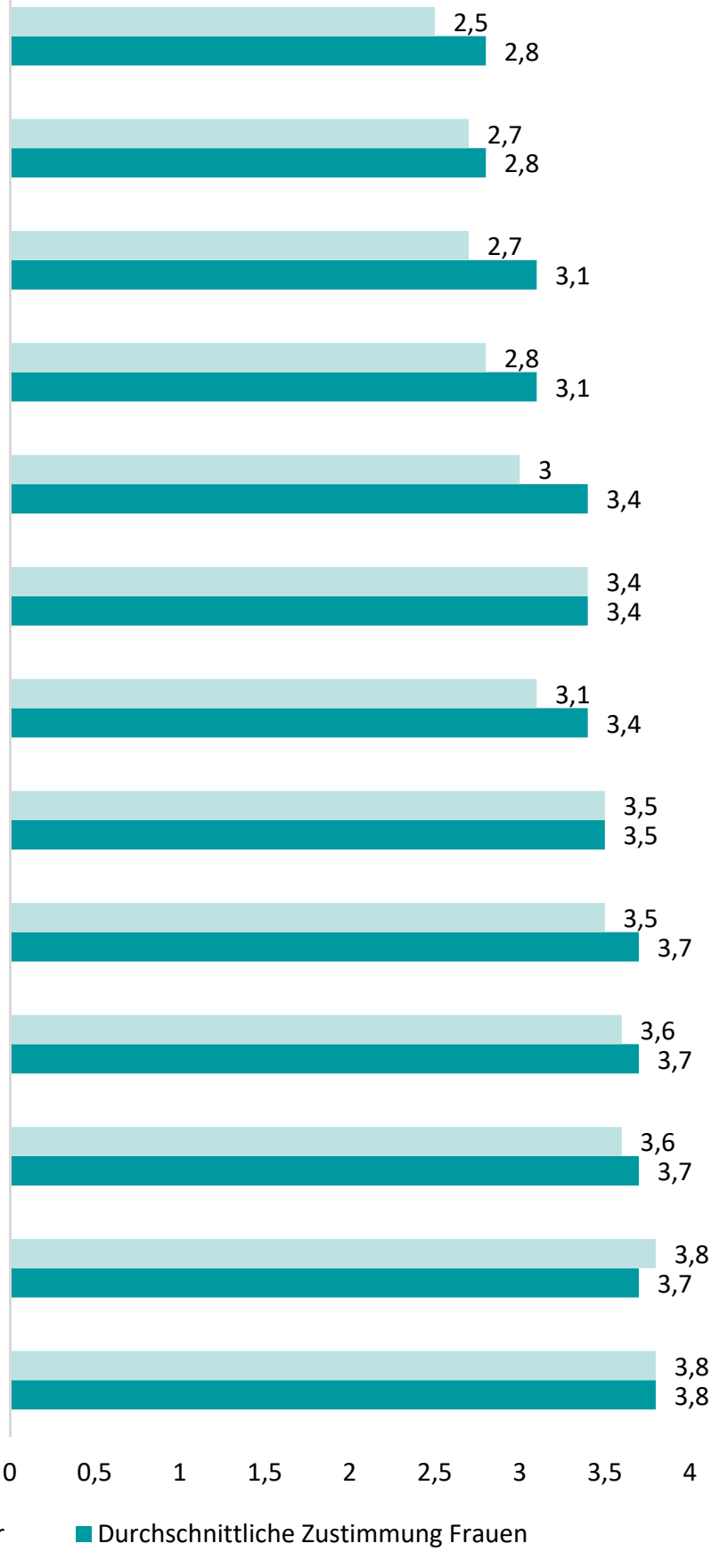

Legende: Skala von 0 („Stimme nicht zu“) bis 4 („Stimme sehr zu“); n=Anzahl der Antworten gesamt (Quelle: EvaSys Ergebnisbericht, eigene Darstellung)

Abbildung 8: Persönliche Integration im Fachbereich

Die Frage der Integration, also dem Gefühl zusammenzugehören, wurde insgesamt auch wieder positiv bewertet, wobei Professorinnen und Professoren sich eher ihrer Fächerkultur zugehörig fühlten, als der Hochschule insgesamt. 
Professorinnen fühlten sich in Prozesse der Problemlösung und Entscheidungsfindung im Fachbereich stärker einbezogen, als ihre männlichen Kollegen Während 38,1 Prozent der Professorinnen der Aussage sehr zustimmten, sind es bei den Professoren nur 21,5 Prozent. 42,9 Prozent der antwortenden Professorinnen stimmten der Aussage, Ich habe eine Stimme im Prozess, wie die Ressourcen verteilt werden' sehr zu, während es bei den Professoren nur 18,5 Prozent sind (Abbildung 9).

\section{Teilhabe im Fachbereich}

Ich habe eine Stimme im Prozess wie Ressourcen zugeteilt/verteilt werden. $(n=86)$

Ich fühle mich bei Prozessen der Problemlösung und Entscheidungsfindung im Fachbereich voll einbezogen. $(n=86)$

\[ \begin{array}{rlrllllll}0 & 0,5 & 1 & 1,5 & 2 & 2,5 & 3 & 3,5 & 4\end{array} \]
Durchschnittliche Zustimmung Männer $\quad$ Durchschnittliche Zustimmung Frauen

Legende: Skala von 0 („Stimme nicht zu“) bis 4 („Stimme sehr zu“); n=Anzahl der Antworten gesamt (Quelle: EvaSys Ergebnisbericht, eigene Darstellung)

Abbildung 9: Teilhabe an Entscheidungsprozessen im Fachbereich

Sowohl in Bezug auf die Einbindung in Entscheidungsprozesse als auch die Antwort auf die Frage danach, ob die eigene Sichtweise im Fachbereich geäußert werden darf, gab es keinen signifikanten Geschlechtsunterschied. Professor*innen waren zufrieden mit ihren Führungskräften und mit der Wahrnehmung ihrer Meinungen in Fachbereichstreffen. Auch wenn die Bewertungen $\mathrm{zu}$ informellen und nicht im direkten Kontakt liegenden Interaktionen etwas schlechter ausfielen, kann die Integration in den Fachbereichen von beiden Geschlechtern als gut interpretiert werden. Grundsätzlich fühlten sich Professorinnen wie Professoren sehr respektvoll behandelt, egal ob von ihren Vorgesetzten, den Studierenden, den Mitarbeitenden im Fachbereich oder von Gleichgestellten.

\subsubsection{Fachbereichsmaßnahmen zur Gewinnung von Frauen in Professuren}

Generell war die Zustimmung zu der Aussage, dass es zu wenige Professorinnen im eigenen Fachbereich gab, also mehr Professorinnen berufen werden sollten, bei beiden Geschlechtern hoch. 


\section{Organisationsklima und Karrierechancen für Professorinnen}

Mein Fachbereich besetzt zu wenige Führungspositionen mit Professorinnen. $(n=69)$

Mein Fachbereich hat Schritte unternommen, um das Klima für Frauen zu verbessern. $(n=48)$

In meinem Fachbereich gibt es zu wenige Professorinnen. $(n=76)$

Mein Fachbereich hat sich bemüht, Frauen in Führungspositionen zu befördern. $(n=57)$

Mein Fachbereich hat Wege gefunden, Professorinnen zu rekrutieren. $(n=71)$

Ich kommuniziere Ausschreibungen innerhalb meiner fachlichen Netzwerke an potentielle Kandidatinnen. $(n=63)$

In meinem Fachbereich ist das Klima für Frauen gut. $(n=77)$

Mein Fachbereich hat aktiv Professorinnen geworben. $(n=61)$

Ich spreche gezielt bei Ausschreibungen aus meiner Perspektive geeignete Kandidatinnen an. $(n=64)$

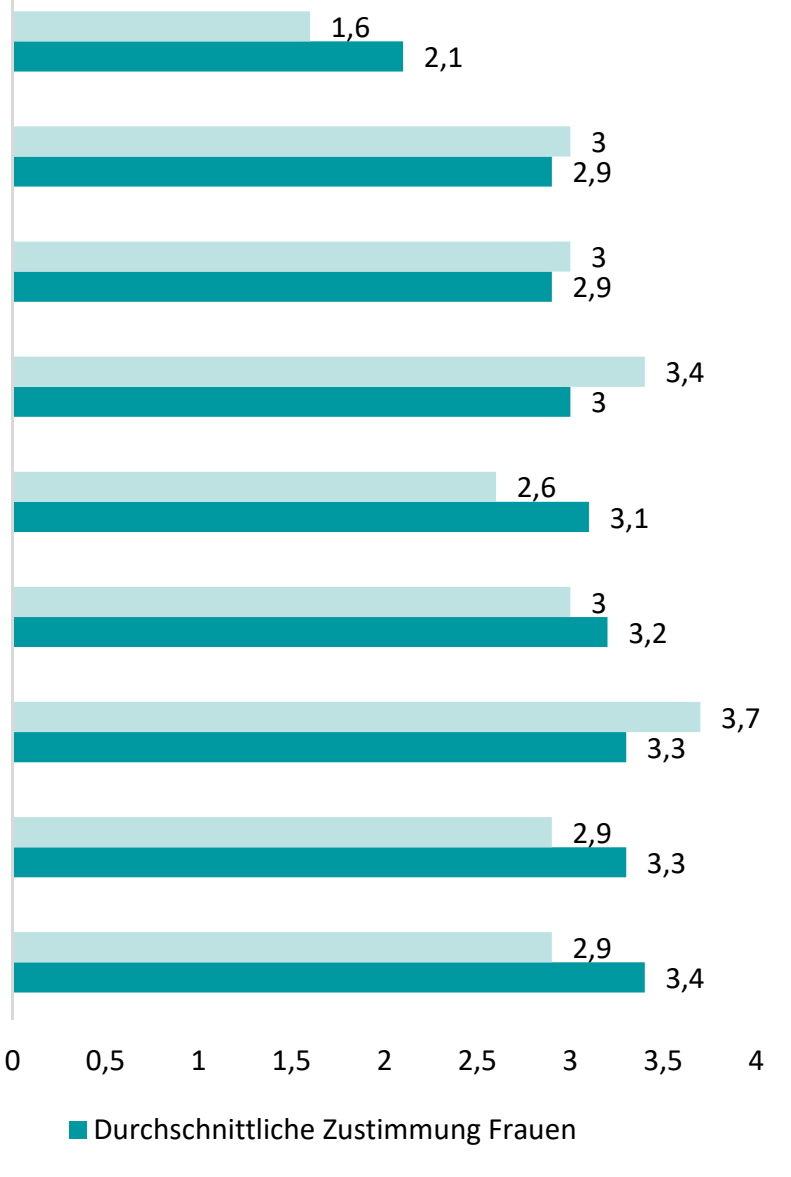

Legende: Skala von 0 („Stimme nicht zu“) bis 4 („Stimme sehr zu“); n=Anzahl der Antworten gesamt (Quelle: EvaSys Ergebnisbericht, eigene Darstellung)

Abbildung 10: Rekrutierung, Klima und Chance auf Führungspositionen von Professorinnen

Zu der offenen Frage, welche Tipps zur Rekrutierung von Frauen gegeben werden, wurden 16 Rückmeldungen gegeben, davon 14 von Männern. Ihre Antworten lassen sich in drei Kategorien zusammenfassen:

- Grundausbildung/ früher ansetzen (Post-Docs, Coachings, Promotionskollege, technisches Studium)

- Ausstattung einer HAW-Professur verbessern, attraktiver machen: Zustand der Hochschulgebäude war abschreckend (Fragebogennr. 30)

- HAW-Professur als Karriere-Option bekannter machen: Viele in der Industrie wissen gar nichts über diese Karriere-Option (Fragebogennr. 43)

Betrachtet man die Gesamtheit aller Aussagen, nutzten die meisten Professor*innen ihr wissenschaftlichen Netzwerke zur Ansprache von Kandidatinnen, um direkt bekannte Frauen anzusprechen. 


\subsubsection{Programme und Ressourcen an der Beuth Hochschule für Technik}

Die verschiedenen Programme und Initiativen der Beuth Hochschule für Technik (s. Abbildung 11) sollten Aufschluss über den Bekanntheitsgrad und den Nutzen bei den Professor*innen geben und dienen dazu, das Verbesserungspotential für zukünftige Unterstützungsmaßnahmen im Berufsalltag zu identifizieren. Die Unterstützungsangebote der Hochschule in Abbildung 11 waren größtenteils und geschlechterübergreifend allen Befragten bekannt. Dabei fiel auf, dass nur wenige Angebote und Maßnahmen auch in Anspruch genommen werden. Von über 20 Prozent der Professorinnen wurden der Pausenexpress, verschiedene Weiterbildungsangebote oder auch das Beuth Handbuch zum Studium in Anspruch genommen.

Die auf den ersten Blick erstaunlich gering anmutende Nutzung familienfreundlicher Angebote, wie das Familienzimmer oder der Kindernotdienst mag darauf zurückzuführen sein, dass die befragten Personen keine Kinder im betreuungsintensiven Alter haben und daher die Angebote der Hochschule für sie nicht mehr relevant sind.

\subsubsection{Work-Life-Balance}

Die antwortenden Professor*innen waren in der Tendenz mit ihrer Work-Life-Balance zufrieden. Die Aussage „Ich bin normalerweise zufrieden mit der Balance zwischen meinem beruflichen und privaten Leben" bewerten die Antwortenden auf einer Skala von 1 = "stimme nicht zu" bis 4 = "stimme sehr zu" durchschnittlich mit einem Wert von 3,1. Die Geschlechter unterschieden sich kaum in ihrer Bewertung. In Bezug auf das Thema Work-Life-Balance fanden sich jedoch Unterschiede zwischen Professorinnen und Professoren, die auch statistisch signifikant waren. Die Professorinnen gaben signifikant häufiger an, dass sie aufgrund persönlicher Verpflichtungen auf berufliche Aktivitäten wie zum Beispiel die Teilnahme an Konferenzen oder die Arbeit an Veröffentlichungen verzichten müssen ( $p=0,014)$. Männer wurden in der Mehrzahl der Fälle weniger in der Karriereentwicklung durch sogenannte Care-Aufgaben behindert, wie beispielsweise die Kinderbetreuung, haushaltsnahe Tätigkeiten, die Betreuung von pflegebedürftigen Angehörigen und stimmten dieser Aussage seltener zu.

Umso erstaunlicher war es, dass die Aussage „Persönliche Verantwortlichkeiten und Engagements haben meine Karriereentwicklung verlangsamt“ von beiden Geschlechtern mit Mittelwerten von 2 bzw. 2,2 sehr ähnlich bewertet wurden. 


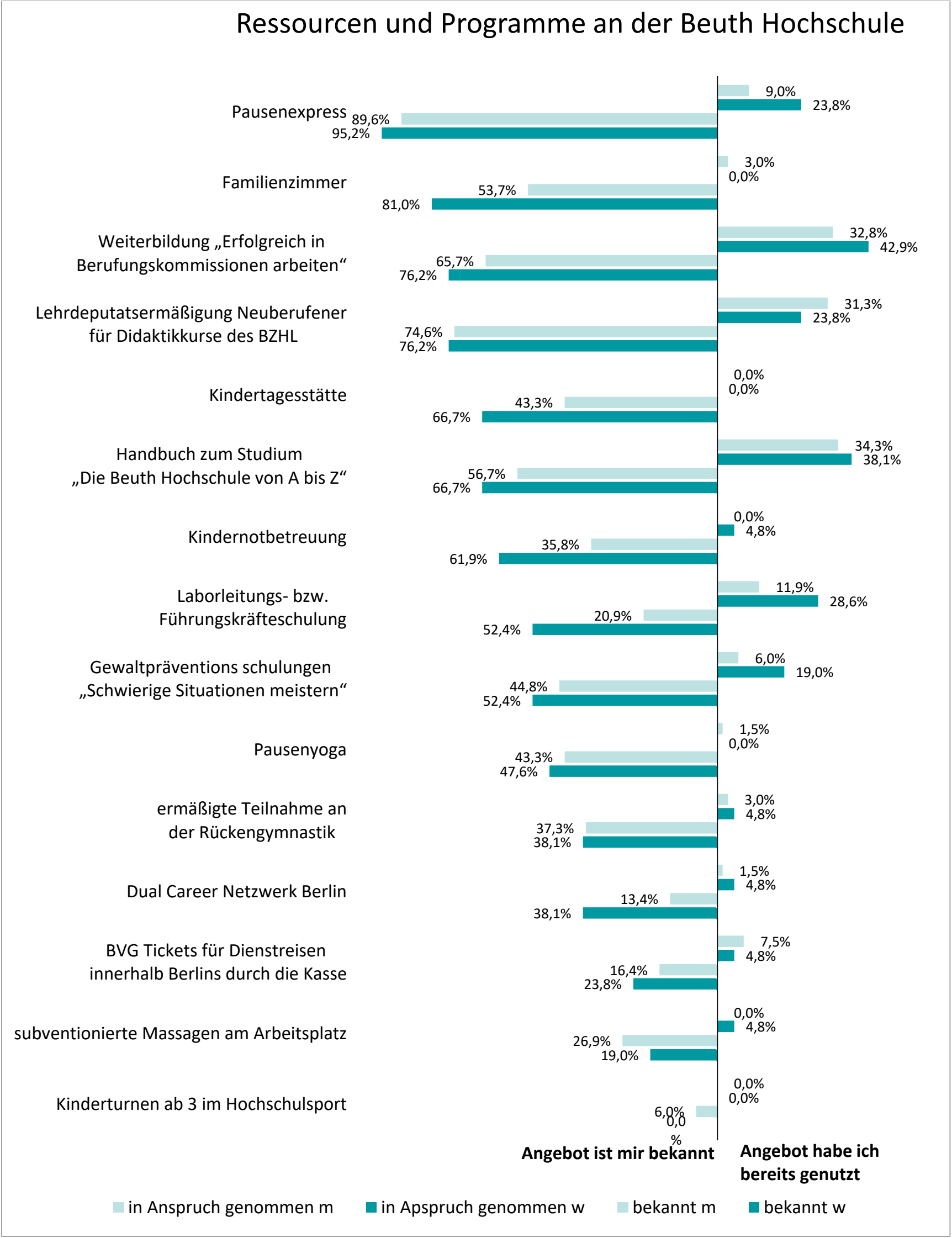

Legende: Angaben in Prozent, getrennt nach männlich $(m, n=75)$ und weiblich $(w, n=21)$

Abbildung 11: Ressourcen und Programme an der Beuth Hochschule 
Obwohl Frauen also aufgrund privater Verpflichtungen auf berufliche Aktivitäten verzichten müssen, haben sie nicht stärker als ihre männlichen Kollegen den Eindruck, dass sich ihre Karriere verlangsamt hatte.

Der zweite signifikante Unterschied liegt in der empfundenen Wichtigkeit der Kinderbetreuung: Professorinnen bewerteten das Vorhandensein einer Kinderbetreuung ( $p=0,002)$, Kindernotbetreuung $(p=0,000)$ und Unterstützung beim Finden als wichtiger $(p=0,000)$, auch wenn sie es selbst, wie unter „Programme und Ressourcen“ (Abbildung 11) dargestellt, kaum nutzten $(p=0,002 ; P=0,000)$. In diesem Fragebogen gaben 75 Prozent an, Mütter zu sein und 63 Prozent Väter, die Gesamtzahlen an der Beuth Hochschule für Technik sind anders herum verteilt: 81 Prozent aller Professoren haben Kinder und nur 66 Prozent aller Professorinnen.

Die Zustimmung zu ausreichender Unterstützung der Hochschule für werdende Eltern bzw. Eltern kleiner Kinder war unter den Frauen gering. Der Mittelwert liegt bei 1,9 (1 = Stimme nicht zu, 4 = Stimme sehr zu) im Gegensatz zu den männlichen Antwortenden, die mit 2,7 eher zustimmen. Keine der antwortenden Professorinnen stimmte dem sehr zu und nur 23 Prozent der Frauen stimmten etwas zu, die Mehrzahl war gegenteiliger Meinung: ganze 43 Prozent stimmten nicht zu. Die Freitext-Kommentare erläuterten hierzu vor allem die Wichtigkeit, die Lehre den Kinderbetreuungszeiten anzupassen, sowohl, was die Ferienzeiten betrifft, als auch mögliche Block- oder Online-Lehre.

Die Hälfte aller Antwortenden war sich einig, dass die Uhrzeiten für Besprechungen im Fachbereich wenig familienfreundlich waren, da sie früh am Morgen oder spät am Abend stattfanden. Professorinnen und Professoren unterscheiden sich in ihrer Bewertung nicht. Die Hochschule als Gesamtes legt ihre Besprechungen etwas familienfreundlicher als die Fachbereiche laut den Antwortenden.

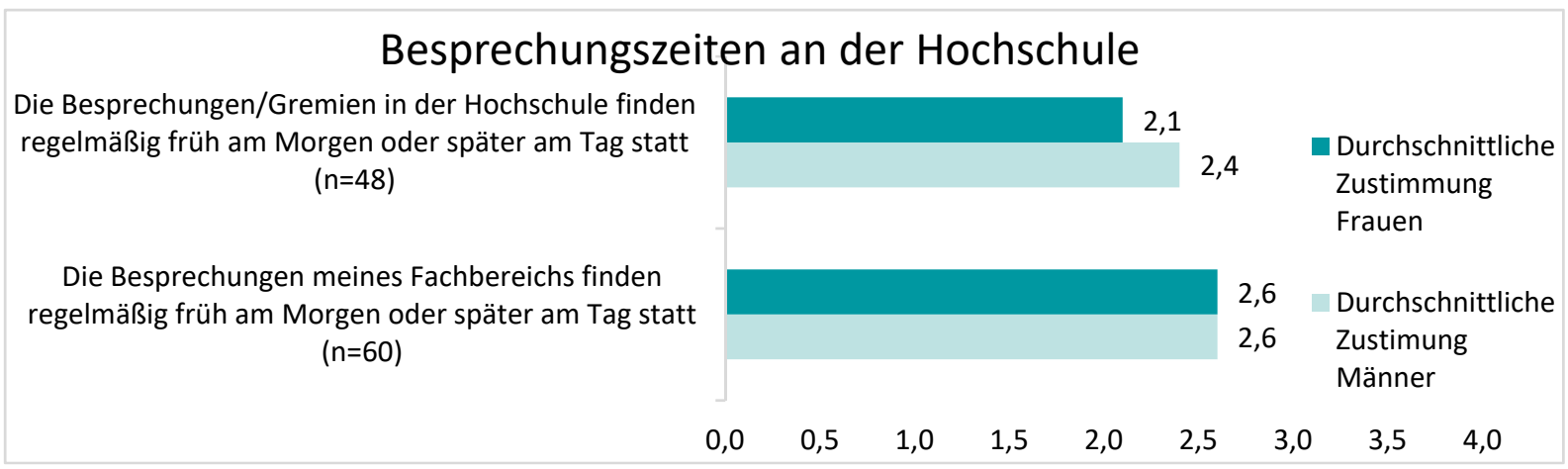

Legende: Skala von 0 („Stimme nicht zu“) bis 4 („Stimme sehr zu“); n=Anzahl der Antworten gesamt (Quelle: EvaSys Ergebnisbericht, eigene Darstellung)

Abbildung 12: Familienfreundlichkeit von Besprechungen im Fachbereich 


\subsubsection{Zufriedenheit}

Die allgemeine Zufriedenheit aller Antwortenden an der Hochschule lag mit einer durchschnittlichen Bewertung von 2,8 im oberen mittleren Bereich. Professorinnen waren jedoch etwas zufriedener (3,0 zu 2,7) und würden die Beuth Hochschule für Technik signifikant ( $p=0,047$ ) häufiger empfehlen, als ihre männlichen Kollegen. Sie hatten außerdem weniger ernsthaft darüber nachgedacht, ihre Arbeitgeberin zu wechseln (siehe Abbildung 13).

\section{Zufriedenheit mit der Hochschule}

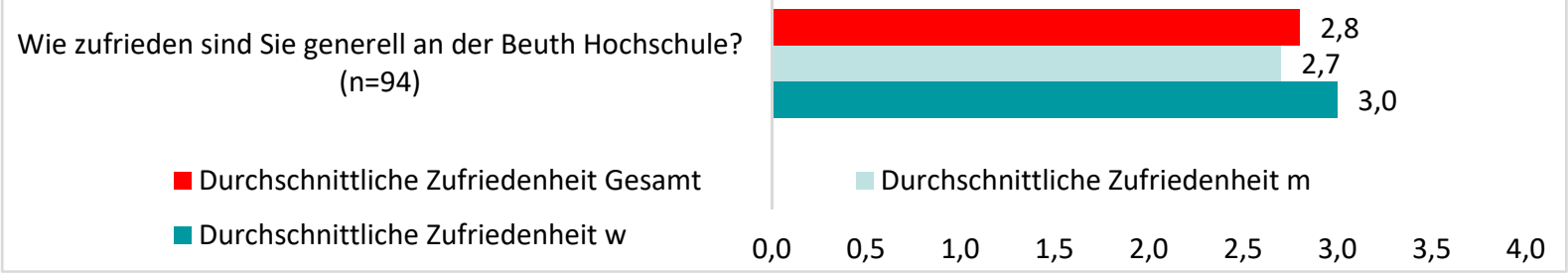

Legende: Skala von 0 („Unzufrieden“) bis 4 („Sehr zufrieden“); n=Anzahl der Antworten gesamt; w=weiblich, m=männlich (Quelle: EvaSys Ergebnisbericht, eigene Darstellung)

Abbildung 13: Generelle Zufriedenheit an der Beuth Hochschule für Technik

Die Zufriedenheit an der Beuth Hochschule deckte sich mit dem Ergebnis aller deutscher Professoren, die zu 70 Prozent zufrieden oder sehr zufrieden mit ihrer Arbeitssituation sind (Sembritzki \& Thiele 2019).

Die Freitext-Kommentare zur Zufriedenheit, zur Unzufriedenheit und zu den Gründen, warum über eine Kündigung nachgedacht wird, wurden mittels MAXQDA ausgewertet und in einer Matrix zusammengefasst, um die für die Professor*innen wichtigsten Punkte übersichtlich darzustellen.

„Nette Kollegen, viele Freiheiten, nette Studierende“ fasste eine Professorin die wesentlichen Punkte zusammen, die die Professor*innen zufrieden machten, die Beuth Hochschule für Technik als Arbeitgeber ausgewählt zu haben. Das Wort „nett“ wurde auch von den Professoren in den Kommentaren häufig gebraucht: „Interessierte Studierende, nette Kolleg*Innen“, ebenso wie die „Gestaltungsfreiräume, Zeitautonomie (...)“. 


Codesystem
Ausstattung
Status Professur
Spaß am Fach/ Freude Fachrichtung
Serwaltungszusammenarbeit, auch Hoc
Studierendenzusammensetzung
Gehalt und Arbeitsmodalitäten
Arbeitsklima des Fachbereiches / Fache
Kolleginnen und Kollegen
Work Life Balance
Möglichkeit zu unterrichten
Snbefristete Tätigkeit
Standortfaktor

Abbildung 14: Codesystem zur Zufriedenheit

(Quelle: MAXQDA, Auswertungsergebnis)

Die Faktoren, die zur Unzufriedenheit beitrugen, fassten die folgenden Ankerzitate einer Professorin und eines Professors zusammen: „Dominanz Verwaltung und dortige Prozesse, Ausstattung Fachbereiche“ und „immenser Administrationsaufwand!!!!“.

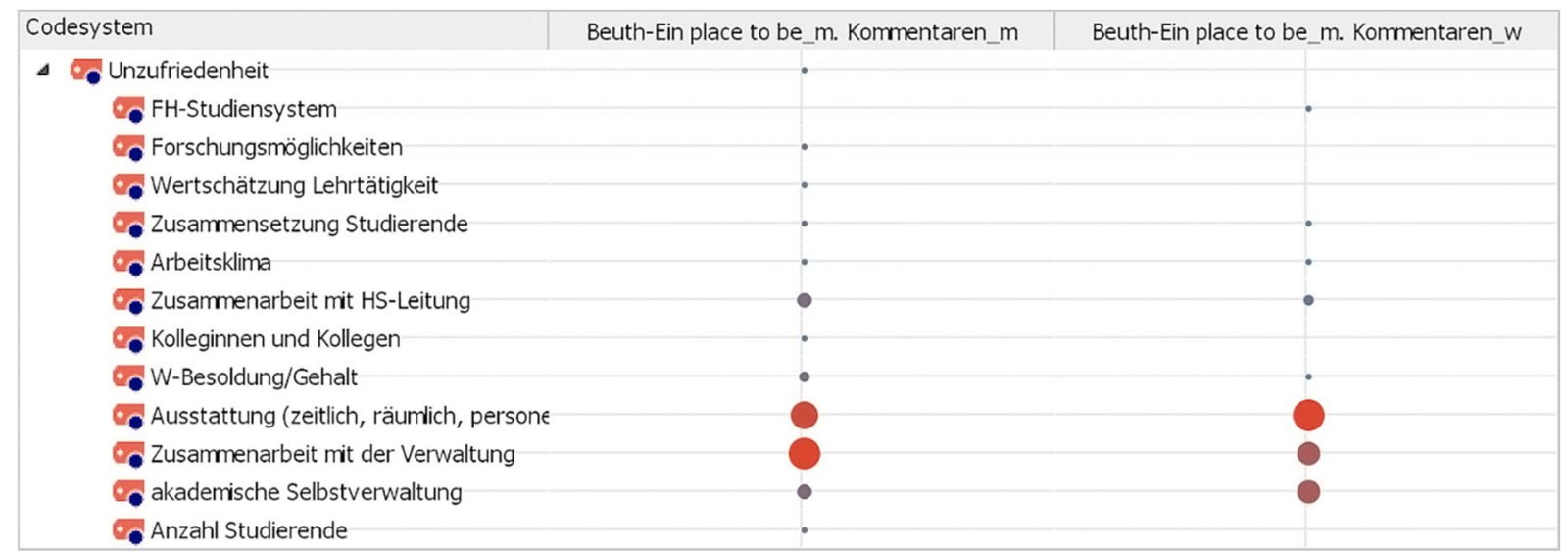

\section{Abbildung 15: Codesystem zur Unzufriedenheit}

(Quelle: MAXQDA, Auswertungsergebnis)

Sowohl die Kommentare zur Instandhaltung, Bauzustand und Raumnot wurden zur (räumlichen) Ausstattung gezählt, als auch jene, die sich beispielsweise über nicht ausreichende (zeitliche oder personelle) Ausstattung bei der Betreuung von Abschlussarbeiten äußerten: „Graduelle schleichende Schlechterstellung in allen Bereichen: Zu viele Bachelor- und Masterarbeiten, denen kaum noch eine Lehrdeputatsermäßigung gegenübersteht". 


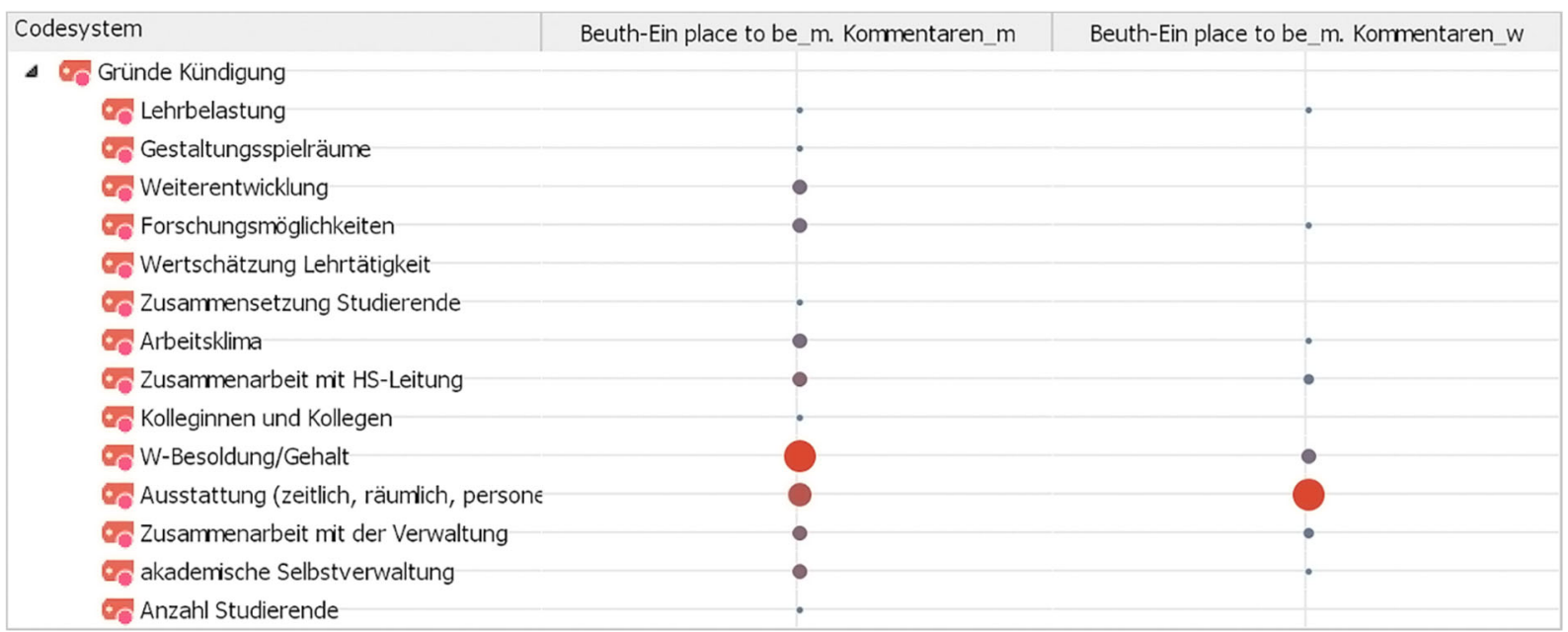

Abbildung 16: Codesystem zu den Kündigungsgründen (Quelle: MAXQDA, Auswertungsergebnis)

Bei den Professorinnen wurde die Ausstattung als Hauptgrund genannt, möglicherweise kündigen zu wollen, das Gehalt, wenn überhaupt, dann im Nebensatz, wie das folgende Ankerzitat illustriert: „Ausstattung der Labore und Lehrräume, Gehalt; Finanzielle und zeitliche Möglichkeiten, Forschung zu gestalten (...)“. Wohingegen die Professoren hauptsächlich die als zu gering empfundene Besoldung nannten und für eine „Aussicht auf Universitätsprofessur mit höherer Besoldung" oder wegen des „Besoldungsverhalten des Berliner Senats“ kündigen würden. Die Gründe für diese Befunde könnten laut der Studie von Sembritzki und Thiele (2019) im vorherigen Einkommen liegen. Aus ihrer Studie ergab sich, dass das ProfessurenEinkommen der Männer, im Vergleich zu den Tätigkeiten vor der Professur, verringert hatte, insbesondere in den MINT-Fächern. Bei den befragten Frauen hat sich das Einkommen jedoch erhöht bzw. blieb weitgehend unverändert. Dieses Ergebnis wird von den Wissenschaftlern als Gender Pay Gap angesehen, der sich durch die Berufung teilweise aufgehoben hat (Schlegel 2006; Schmidt 2016; Sembritzki \& Thiele 2019).

\section{Zusammenfassung und Fazit}

Alle bisherigen Anstrengungen und Maßnahmen haben bislang nicht den gewünschten Erfolg erbracht ein ausgewogenes Größenverhältnis zwischen Professorinnen und Professoren an technischen Hochschulen zu erreichen. Die vorliegende Studie hat beispielhaft für die Beuth Hochschule für Technik untersucht, was HAW im Recruitingprozess und im Bewerbungsverfahren, in den Strukturen und im Organisationsklima verändern sollten, um eine technische Hochschule zu einem attraktiven Arbeitsort (place to be) zu machen. Hierbei lag der Schwerpunkt bei der Identifikation möglicher geschlechterbezogener Unterschiede in den Bewertungen verschiedener Attraktivitätsdimensionen. Dabei zeigte sich, dass der größte signifikante Unterschied zwischen den Geschlechtern in Bereich der Forschung liegt. Professorinnen ist 
es wichtiger als Professoren forschen zu können. Der zweitgrößte signifikante Unterschied lag darin, dass für Männer eine bessere Work-Life-Balance im Vergleich zu der vorherigen Tätigkeit, in der Wirtschaft offensichtlich ein wichtigerer Grund für eine Professur darstellt, als für Frauen. Frauen übernehmen mehr informelle Arbeiten und empfinden ihre Überbelastung in der akademischen Selbstverwaltung durch die Quotierung trotzdem als weniger belastend und wollen sich zukünftig dennoch engagieren.

Die Auswertung hat auch ergeben, dass die befragten Frauen forschungsstärker und glücklicher an der Hochschule sind als ihre männlichen Kollegen und die Hochschule als Arbeitsort stärker weiterempfehlen würden, als ihre männlichen Kollegen.

Deutliche Unterschiede zeigten sich auch bei der Bewertung der Karriereentwicklung. Professorinnen gaben häufiger an, dass ihre persönlichen Verpflichtungen und Care-Aufgaben sie an beruflichen Aktivitäten hinderten. Folglich ist für Frauen auch die Unterstützung bei Familienarbeit wichtiger, als für Männer. Mit Blick auf die potentielle Behinderung der Karriereentwicklung aufgrund von Care-Aufgaben, zeigte sich aber, dass Professorinnen nicht der Meinung sind, dass ihre Karriere dadurch beeinflusst wurde. Dieses Ergebnis kann möglicherweise ein Stichprobeneffekt sein, da es sich bei den Befragten um diejenigen handelt, die ihre Karriere zur Professur erfolgreich abgeschlossen haben.

Betrachtet man die Zufriedenheit und die überwiegend im positiven Bereich liegenden Antworten der Auswertung, dann ist die Grundfrage „Ist die Beuth Hochschule für Technik ein place to be?" klar und geschlechtsunabhängig mit einem „Ja“ zu beantworten.

\section{Maßnahmen und Lösungsansätze}

Als Lösungsansätze für die Bearbeitung der aufgezeigten Problemfelder werden im wissenschaftlichen Diskurs überwiegend formal-organisatorische Maßnahmen vorgeschlagen. So etwa die Einführung von Quoten für Frauen bei der Besetzung der Berufungskommissionen, ein gezieltes Gendercoaching der in die Auswahlprozesse einbezogenen Personen, um deren Sensibilität für das Thema zu steigern (Steinweg 2015), die Beschreitung neuer, insbesondere auch Wissenschaftlerinnen berücksichtigender Wege zur Akquisition von Hochschullehrer*innen, Teilanonymisierung von schriftlichen Informationen, um den Einfluss von Gendervorurteilen zu reduzieren (Braun et al. 2015) und die Schaffung von hochschulweiten Berufungsstandards (Jeschke et al. 2013), um nur einige zu nennen. Seitens der Bundeskonferenz der Frauen- und Gleichstellungsbeauftragten an Hochschulen (BuKoF e. V.) wird besonders dem Instrument der Standardisierung große Bedeutung beigemessen. In einer Stellungnahme zur öffentlichen Anhörung des Bundestagsausschusses für Bildung, Forschung und Technik vom 19. Oktober 2016, die dem Thema „Chancengleichheit im Wissenschaftssystem“ gewidmet 
war, wird für alle Gesetzesvorhaben und Förderprogramme die Berücksichtigung von Gleichstellungsstandards im personellen und strukturellen Bereich sowie hinsichtlich der Inhalte, Kompetenzen und Ressourcen gefordert (Niehoff 2016).

Die Richtung notwendiger Veränderungen und mögliche Maßnahmengebiete zur Überwindung der Benachteiligung von Frauen innerhalb des Systems, Wissenschaft' sind also durchaus klar umrissen, wobei sie wesentlich auf die Organisationsstrukturen bezogen sind. Der dahinterstehende Gedanke ist, durch gezielte Eingriffe in diese Strukturen Bedingungen zu schaffen, die zu mehr Chancengleichheit der Geschlechter und damit auch zu mehr Vielfalt in Forschung und Lehre führen, und zwar beruhend auf der Annahme, dass sich daraus zwangsläufig auch eine Veränderung der Organisationskultur ergibt. Die Vorstellung einer auf diese Art provozierten positiven Entwicklung ist sicher nicht falsch. Es stellt sich allerdings die Frage, unter welchen Bedingungen sie sich am effektivsten und damit letztlich auch am effizientesten für die Organisation, wie auch die jeweiligen Betroffenen vollziehen kann.

Zur Umsetzung von Reformen können basierend auf der vorliegenden Studie folgende erste Handlungsempfehlungen für die Beuth Hochschule gegeben werden. Sie zielen darauf ab, stereotype Genderkategorien und weitere soziale Differenzkategorien in der Organisationsstruktur und -kultur zu reflektieren, zu dekonstruieren und damit aufzubrechen (Rheinländer 2015).

\section{Active Sourcing: Gezielte Ansprache von Frauen}

Die Akquirierung von Professor*innen durch adäquate Ausschreibungen und direkte Kontaktaufnahme ist ein wichtiger Schritt einer zielgruppendifferenzierten Personalrekrutierung. Dabei kommt dem ,Active Sourcing' eine besondere Bedeutung zu.

Active Sourcing bedeutet im Wesentlichen frühzeitig und proaktiv auf potentiell geeignete Kandidatinnen z. B. auf Tagungen und Kongressen sowie in relevanten Netzwerken zu zugehen, sie über mögliche Karrierewege an der Hochschule zu informieren und die Hochschule als einen attraktiven Arbeitsort (place to be) zu präsentieren. Die Auswertung zeigt, dass ein Großteil der Professor*innen diese Aufgabe nicht wahrnimmt oder sich der Wichtigkeit nicht bewusst ist. Dementsprechend sollte die Bedeutung eines Active Sourcing hochschulweit stärker in den Blick genommen werden z. B. im Rahmen der Schulungsmaßnahmen für Berufungsmitglieder. Da diese Schulungsmaßnahmen aber in der Regel erst besucht werden, wenn eine konkrete Berufung ansteht, ist der Schulungszeitpunkt für ein Active Sourcing im jeweiligen konkreten Berufungsverfahren meist zu spät, denn es setzt weit früher an. Hilfreich könnte es daher sein, verpflichtende Schulungen für alle Professor*innen zum Thema Active Sourcing und Gestaltung gendersensibler Berufungsverfahren anzubieten. Erleichternd könnten hier auch online Schulungs- und Informationsangebote sein. 
Sollte sich der gefundene Geschlechtsunterschied hinsichtlich der Wichtigkeit der Forschungsmöglichkeiten bestätigen, sollte auch bei der Akquise von Professorinnen besonders auf Forschungsmöglichkeiten und Forschungsunterstützung hingewiesen werden.

\section{Personalentwicklung und Diversity Management}

Laut des Wissenschaftsrats (2018) ist die systematische Herangehensweise zum Thema Personalentwicklung, abgesehen von der akademischen Weiterqualifizierung, für den Hochschulsektor noch relativ neu. Hier gilt es neue organisationale Strukturen aufzubauen, die Personalentwicklung und Diversity Management miteinander verbinden.

Zu einer attraktiven Personalentwicklung gehört für Professor*innen an HAW der Austausch mit der betrieblichen Praxis. Dieser kann im Kontext von gemeinsamen Forschungsvorhaben erfolgen oder durch Freistellungen zur Auffrischung des Praxiswissens. Aktuell werden aber laut Wissenschaftsrates an technischen Hochschulen Forschungsfragen bzw. außerhochschulische Praxisphasen mehrheitlich nicht in Anspruch genommen, weil die Lehrdeputate in MINT-Fächern häufig nicht aufgefangen werden können (Wissenschaftsrat 2018). Hier besteht dringender Regelungsbedarf, der jedoch nicht nur innerhalb einzelner Hochschulen, sondern regional oder bundesweit vorgenommen werden muss.

\section{Gendergerechtes Organisationsklima}

Der wesentliche Kritikpunkt der Professor*innen in der vorliegenden Umfrage lag in den als langsam und hinderlich wahrgenommenen Verwaltungsabläufen. Hier empfiehlt sich eine Professionalisierung und Weiterentwicklung der Verwaltungsprozesse hin zu mehr Service-Orientierung.

Zu einem gendergerechten Organisationsklima gehören Möglichkeiten der Beteiligung und Entscheidungstransparenz auf verschiedenen Ebenen der Organisation. Auf der Ebene der Fachbereiche sind die Beurteilungen der Befragten tendenziell positiv. Dieses Ergebnis sollte Grund und Ansporn sein, diese positiven Beurteilungen zu bewahren und zu verstetigen. Mittlere Beurteilungen bei den Fragen „Ich fühle mich in informelle Netzwerke in meinem Fachbereich integriert" oder "ich mache Arbeiten, die in meinem Fachbereich nicht formal anerkannt werden“ und „ich muss Aufgaben machen, die nicht zu meinem Aufgabenverständnis gehören“, liefern konkrete Hinweise, wo die Verbesserungspotentiale liegen.

Interessant sind hier auch die Hinweise auf Abweichungen der realen Arbeitszeitverteilungen in Bezug auf Lehre, Forschung, Beratung, akademische Selbstverwaltung und der gewünschten Verteilungen. Beide Geschlechter möchten den Zeitanteil für Forschung auf Kosten der Lehre deutlich erhöhen. Hier sollte gerade unter dem Gesichtspunkt einer innovativen Organisationsentwicklung nachgedacht werden, wie diesen Wünschen nach mehr Forschung angemessen Rechnung getragen werden kann. 
Kritischer werden die verfügbaren technischen, räumlichen und personellen Ressourcen beurteilt. Hier sollte ebenfalls im Sinne der Organisationsentwicklung überlegt werden, wie langfristig bessere Ressourcenzuschnitte sichergestellt werden können.

\section{Organisationsklimaanalyse als kontinuierliches Organisationsentwicklungsinstrument etablieren}

Zuletzt ist eine regelmäßige Wiederholung der Befragung sinnvoll. Die Wahrnehmung jener Zustände, Erscheinungen, Setzungen und aus ihnen resultierenden Haltungen, die als ,Organisationskultur' bezeichnet werden, hat für das Funktionieren der jeweiligen Organisation eine hohe Relevanz. Die Ergebnisse der Studie lassen Tendenzen zu Handlungsempfehlungen und mögliche Maßnahmen zu, die in zukünftigen Untersuchungen auf ihre Umsetzungserfolge analysiert werden müssten.

So kann aus vorhergehenden Befragungen ein Erwartungsraster entwickelt werden, auf dessen Grundlage dann die Erfüllung unterschiedlicher Standards verschiedener Merkmale des Organisationsklimas durch Wiederholungsmessungen bewertet werden. Es wäre damit ein Werkzeug geschaffen, um die Exzellenz von Hochschulen bezüglich Gendergerechtigkeit tatsächlich zu erfassen und sie nicht nur anhand von Daten, wie dem Anteil an Doktorandinnen und Fachartikeln oder der Zahl an Projekten und der Summe eingeworbener Drittmittel zu definieren.

Die Organisationskultur ist ohne Zweifel ein qualitativer Faktor, der letzten Endes die Wirksamkeit der Organisation wesentlich mit beeinflusst und die Brücke zur Auseinandersetzung mit den rein personenbezogenen Faktoren des Systems, Wissenschaft' bildet. An dieser Stelle sollte zukünftige Forschung ansetzen. Es gilt, einerseits weitere Informationen und Daten über die bestehenden Strukturen zu sammeln, um sie bis ins Detail analysieren und verstehen zu können, andererseits die Wirkung dieser Strukturen und möglicher Veränderungen auf die Beteiligten abschätzen und/ oder nachvollziehen zu können, nicht zuletzt vor dem Hintergrund persönlicher Erwartungen und Erfahrungen der Protagonist*innen.

Der Publikation liefert am Beispiel der Beuth Hochschule für Technik einen Einblick in das Innenleben einer technisch ausgerichteten Hochschule. Es werden darüber Informationen geliefert, was die wichtigsten Entscheidungsfaktoren für eine Bewerbung an der Hochschule waren, wie das Bewerbungsverfahren und wie das Organisationsklima erlebt wird. Damit werden Hinweise auf mögliche Maßnahmen für geschlechtergerechte Berufungsverfahren und ein geschlechtergerechtes Organisationsklima gegeben.

Die Aussagekraft dieser Studie ist durch die Spezifität der Stichprobe und die geringe Fallzahl, beschränkt. Die Ergebnisse können daher nur als Tendenzbeschreibung gewertet werden. Es bedarf weitere Daten und Studien in diesem Feld, um die Frage, was eine geschlechterge- 
rechte technisch ausgerichtete Hochschule ausmacht, zu beantworten. Besondere Aufmerksamkeit könnte zum einen auf der individuellen Ebene der Pfadabhängigkeiten der wissenschaftlichen Karrierewege von Frauen an technisch ausgerichteten Hochschulen geschenkt werden. Laut Sembritzki und Thiele (2019) ist dieser Bereich, insbesondere der Pfad der auBerakademischen Berufspraxis noch immer ein undurchsichtiger Bereich. Der Wissenschaftsrat (2018) hebt hervor, dass zukünftig neben den Berufungsverfahren auch neue soziale Innovationen, Instrumente und Struktur-Reformen in Hinblick auf ihr Attraktivitätspotential für Professor*innen analysiert werden müssen. Zukünftig bedarf es weiterer und vertiefte Analysen über strukturelle Reform der Personalgewinnung, -struktur und -entwicklung an technisch ausgerichteten Hochschulen. 


\section{Abbildungen}

Abbildung 1: Frauenanteil an den Professuren in den MINT-Fächergruppen.

Abbildung 2: Frauenanteile in Prozent in den verschiedenen Qualifikationsstufen der MINT-

Fächer

Abbildung 3: Direkte Aufforderung zur Bewerbung an der Beuth Hochschule......................20

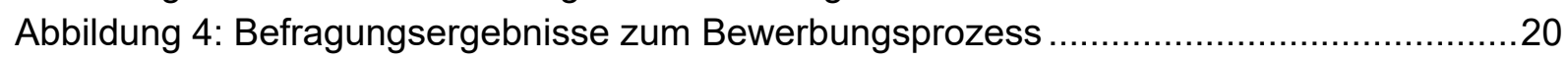

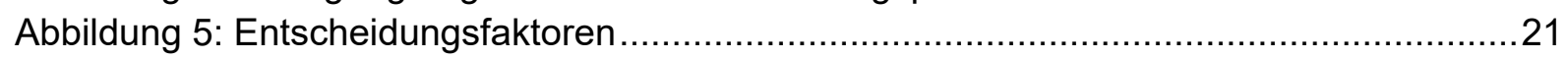

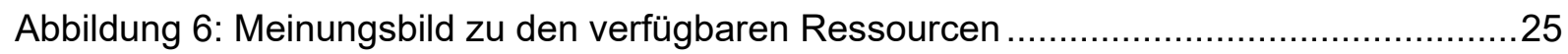

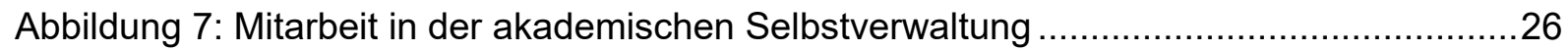

Abbildung 8: Persönliche Integration im Fachbereich ................................................... 27

Abbildung 9: Teilhabe an Entscheidungsprozessen im Fachbereich..................................28

Abbildung 10: Rekrutierung, Klima und Chance auf Führungspositionen von

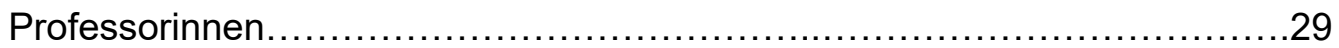

Abbildung 11: Ressourcen und Programme an der Beuth Hochschule .................................31

Abbildung 12: Familienfreundlichkeit von Besprechungen im Fachbereich ..........................32

Abbildung 13: Generelle Zufriedenheit an der Beuth Hochschule für Technik.......................33

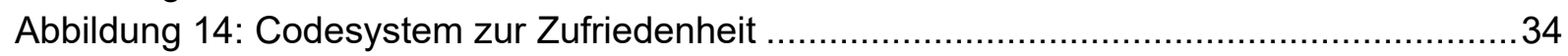

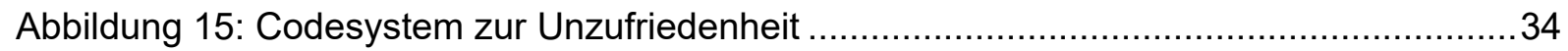

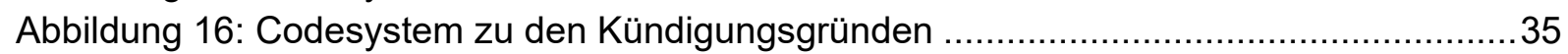

\section{Tabellen}

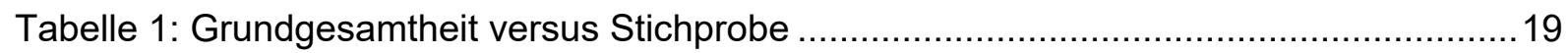

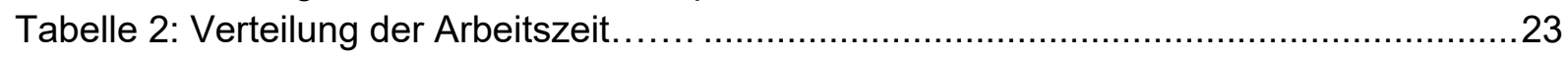

Tabelle 3: Zusammenhang zwischen Zufriedenheit und Verteilung der tatsächlichen

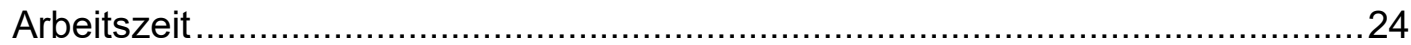

Tabelle 4: Zusammenhang zwischen Zufriedenheit und Verteilung der gewünschten

Arbeitszeit.

Tabelle 5: Differenzwerte zwischen tatsächlicher und gewünschter Arbeitszeit von zufriedenen und weniger zufriedenen Professor ${ }^{\star} I n n e n$ 


\section{Literaturverzeichnis}

Babitsch, B., Ducki, A. \& Maschewsky-Schneider, U. (2012). Geschlecht und Gesundheit. In: K. Hurrelmann \& O. Razum (Hrsg.), Handbuch Gesundheitswissenschaften. Weinheim: Belz Juventa: S. 639-660.

Banaji, M. \& Greenwald, A. (2013). Blindspot: Hidden biases of good people. New York: Delacorte Press.

Berliner Programm zur Förderung der Chancengleichheit für Frauen in Forschung und Lehre (BCP) (2019). Förderbeispiele.

Beaufays, S. (2012). Führungspositionen in der Wissenschaft. Zur Ausbildung männlicher Soziabilitätsregime am Beispiel von Exzellenzeinrichtungen. In: Beaufays, S., Engels, A. \& Kahlert, H. (Hrsg.): Einfach Spitze? Neue Geschlechterperspektiven auf Karrieren in der Wissenschaft. 1. Aufl. Frankfurt / New York: Campus: S. 87-117.

Borgwardt, A. (2013). Arbeitsplatz Hochschule: Aufstieg oder Sackgasse? Berlin: FriedrichEbert-Stiftung.

Bornmann, L., Mutz, R. \& Daniel, H. (2007). Gender differences in grant peer review: A metaanalysis. In: Journal of Informetrics 1 (3): S. 226-238.

Braun, S., Hentschel, T., Peus, C. \& Frey, D. (2015). Chancengleichheit durch professionelle Ressourcenauswahl in der Wissenschaft. In: Peus, C., Braun, S., Hentschel, T., Frey, D. (Hrsg.). Personalauswahl in der Wissenschaft. Evidenzbasierte Methoden und Impulse für die Praxis. Heidelberg: Springer: S. 30-48.

Bundesministerium der Justiz (BMJ) (Hrsg.) (2012). Bundesanzeiger BAnz AT 27.12.2012 B7: Bundesministerium für Bildung und Forschung: Bekanntmachung über die Verlängerung der Bund-Länder-Vereinbarung gemäß Artikel 91b Absatz 1 Nummer 2 des Grundgesetzes über die Fortsetzung des Professorinnenprogramms des Bundes und der Länder zur Förderung der Gleichstellung von Frauen und Männern in der Wissenschaft und Forschung an deutschen Hochschulen (Professorinnenprogramm II). Berlin: Bundesministerium der Justiz.

Bundesministerium für Bildung und Forschung (Hrsg.) (2004). Die Fachhochschulen in Deutschland. Bonn: Bundesministerium für Bildung und Forschung.

Devine P.G., Forscher, P.S., Cox, W.T.L, Kaatz, A. \& Sheridan, J. (2017). A gender bias habitbreaking intervention led to increased hiring of female faculty in STEMM departments. In: Journal of Experimental Social Psychology. 73: S. 211-215.

Färber, C. \& Riedler, U. (2016). Black Box Berufung: Strategien auf dem Weg zur Professur. Frankfurt/Main, New York: Campus.

Färber, C. \& Spangenberg, U. (2008). Wie werden Professuren besetzt? Chancengleichheit in Berufungsverfahren. Frankfurt/Main: Campus.

Felfe, J. (2019). Organisationsdiagnose. In: Schuler, H. \& Moser, K. (Hrsg.). Lehrbuch Organisationspsychologie. 6. Auflage. Bern: Hogrefe: S. 347 -379.

Fuchs, J. \& Maschewsky-Schneider, U. (2002). Geschlechtsangemessene Publikationspraxis in den Gesundheitswissenschaften im deutschsprachigen Raum? -Ergebnisse einer Literaturreview. In: Das Gesundheitswesen. 64 (5): S. 284-291. 
Geißler, R. (2004). Die Illusion der Chancengleichheit im Bildungssystem - von PISA gestört. In: Zeitschrift für Soziologie der Erziehung und Sozialisation (ZSE). 4/2004: S. 362-380.

Gemeinsame Wissenschaftskonferenz (GWK) (Hrsg.) (2017). Chancengleichheit in Wissenschaft und Forschung. 21. Fortschreibung des Datenmaterials (2015/2016) zu Frauen in Hochschulen und außerhochschulischen Forschungseinrichtungen. Heft 55, Materialien der GWK. Bonn. Zugriff am 07.05.2020 unter www.gwk-bonn.de/fileadmin/Redaktion/Dokumente/ Papers/GWK-Heft-55-Chancengleichheit.pdf.

Gemeinsame Wissenschaftskonferenz (GWK) (Hrsg.) (2018). Chancengleichheit in Wissenschaft und Forschung. 22. Fortschreibung des Datenmaterials (2016/ 2017) zu Frauen in Hochschulen und außerhochschulischen Forschungseinrichtungen. Bonn: Gemeinsame Wissenschaftskonferenz (GWK). Zugriff am 07.05.2020 unter https://www.gwk-bonn.de/ fileadmin/Redaktion/Dokumente/Papers/22._FS_Frauenbericht_2018_Heft_60.pdf.

Heusgen, K. (2016). Inter- und intrasektorale Mobilität von Wissenschaftlerinnen und Wissenschaftlern. In: Metz-Göckel, S., Schürmann, R., Heusgen, K. \& Selent, P. (Hrsg.). Faszination Wissenschaft und passagere Beschäftigung. Eine Untersuchung zum Drop-Out aus der Universität. Opladen: Verlag Barbara Budrich: S. 101-120.

Ihsen, S. (2007). Gender und Diversity in Ingenieurwissenschaften. In: Leicht-Scholten, C. (Hrsg.). Gender and Science: Perspektiven in den Natur- und Ingenieurwissenschaften. Bielefeld: transcript Verlag: S. 157-170.

Ihsen, S. \& Ducki, A. (Hrsg.) (2012). Gender Tool Box. Berlin: Beuth Hochschule für Technik Berlin, Schriftenreihe des Gender und Technik-Zentrums (GuTZ).

Isaac, C., Lee, B. \& Carnes, M. (2009). Interventions that affect gender bias in hiring: a systematic review. Academic medicine: journal of the Association of American Medical Colleges 84 (10): S. 1440-1446.

Janssen, J. \& Laatz, W. (2017). Statistische Datenanalyse mit SPSS. Berlin: Springer Gabler: S. $652-657$.

Jeschke, S., Ihsen, S., Vossen, R., Borowski, E., Müller, L., Jeanrenaud, Y. \& Scheibl, K. (Hrsg.) (2013). Projektübersicht und Handreichung zum Verbundvorhaben Genderation BeSt. Untersuchung von geschlechterneutralen und gendersensiblen Berufungsstrategien. Themenschwerpunkt „Frauen an die Spitze“ - Förderbereich des Bundesministeriums für Bildung und Forschung "Strategien zur Durchsetzung von Chancengleichheit für Frauen in Bildung und Forschung". Aachen: Zentrum für Lern- und Wissensmanagement.

Jung, S. (2011). Unbefristet, perspektivenreich, frauenfreundlich? Karrieren im Wissenschaftssystem und ihre Gender-Bias. In: Hempele, A., Keller, A. \& Ortmann, A. (Hrsg.). Traumjob Wissenschaft? Bielefeld: Bertelsmann Verlag: S. 85-90.

Junghans, L. (2012). Die Berufung von ProfessorInnen. Das geschlechtergerechte Berufungsverfahren und seine gerichtliche Überprüfung. GENDER. Zeitschrift für Geschlecht, Kultur und Gesellschaft. 4(1): S. 141-148.

Kanter, R. (1977). Men and Women of the Corporation. New York: Basic Books.

Kauffeld, S. (2019). Organisationsentwicklung. In: Schuler, Heinz. \& Moser, K. (Hrsg.). Lehrbuch Organisationspsychologie. 6. Auflage. Bern: Hogrefe: S. 386-422. 
Kortendiek, B., Hilgemann, M., Niegel, J. \& Hendrix, U. (2014). Gender-Report 2013. Geschlechter(un)gerechtigkeit an nordrhein-westfälischen Hochschulen. Hochschulentwicklungen, Gleichstellungspraktiken, Wissenschaftskarrieren. Zugriff am 22.08.2019 unter www.genderreport-hochschulen.nrw.de.

Kunadt, S., Lipinsky, A., Löther, A., Steinweg, N. \& Vollmer, L. (2014). Gender in der Hochschulforschung. Status Quo und Perspektiven. In: Die Hochschule: Journal für Wissenschaft und Bildung. 23 (1): S. 106-117.

Leicht-Scholten, C., \& Wolffram, A. (2010). Managing Gender and Diversity Mainstreaming an Hochschulen im Spannungsfeld zwischen Theorie und Praxis. GENDER - Zeitschrift für Geschlecht, Kultur und Gesellschaft. 2(2): S. 87-95.

Liebig, B. \& Meyerhofer, U. (2009). Gelebte Gender-Kompetenz. Zugriff am 10.09.2019 unter www.fhnw.ch/ueber-uns/gleichstellung/gut-zu-wissen/download.

Lind, I. (2006). Kurzexpertise zum Themenfeld Frauen in Wissenschaft und Forschung. Bonn: Informationscenter Sozialwissenschaften/CEWS.

Marsh Herbert, W., Bornmann, L., Mutz, R., Daniel, H. \& O’Mara, A. (2009). Gender Traditional and Multilevel Approaches. In: Review of Educational Research. 79 (3): S. 1290-1326.

Metz-Göckel, S., Schürmann, R., Heusgen, K. \& Selent, P. (2016). Faszination Wissenschaft. Passagere Beschäftigte im Spannungsfeld von Hingabe und Aufgabe. Opladen: Verlag Barbara Budrich.

Möller, C. (2015). Herkunft zählt (fast) immer. Soziale Ungleichheiten unter Universitätsprofessorinnen und -professoren. Weinheim/ Basel: Beltz Juventa.

Molvaer, J. \& Stein, K. (1994). Ingenieurin - warum nicht? Berufsbild und Berufsmotivation von zukünftigen Ingenieurinnen und Ingenieuren. Ein interkultureller Vergleich. Frankfurt a.M./New York: Campus Verlag.

Moss-Racusin, C., Dovidio, J., Brescoll, V.., Graham, M. \& Handelsman, J. (2012). Science Faculty's subtle gender biases favor male students. In: Proceedings of the National Academy of Science of the United States of America. 109: S. 16474-16479.

Müller, A., Ohms, G. \& Dittrich, M. (2015). Frauen und MINT: Erkenntnisse zu Gender und Diversity am Beispiel hybrider Lehr-Lernarrangements mit Berufsrückkehrerinnen. In: Rheinländer, K. (Hrsg.). Ungleichheitssensible Hochschullehre. Positionen, Voraussetzungen, Perspektiven. Wiesbaden: Springer Verlag: S.125-140.

Niehoff, A. (2016). Stellungnahme der Bundeskonferenz der Frauen- und Gleichstellungsbeauftragten an Hochschulen (BuKoF) e. V. Öffentliche Anhörung des Bundestagsausschusses für Bildung, Forschung und Technikfolgenabschätzung „Chancengerechtigkeit im Wissenschaftssystem“. Berlin, 19. Oktober 2016. Berlin: BuKoF e. V.

Reuben, E., Sapienza, P. \& Zingales, L. (2014). How stereotypes impair women's careers in science. Proceedings of the National Academy of Sciences of the United States of America. 111: S. 4403-4408.

Rheinländer, K. (2015). Von der Bedeutung und der Möglichkeit einer ungleichheitssensiblen Hochschullehre. In: Rheinländer, K. (Hrsg.). Ungleichheitssensible Hochschullehre. Positionen, Voraussetzungen, Perspektiven. Wiesbaden: Springer Verlag: S. 47-70. 
Samjeske, K. (2012). Gender Bias in der Forschungsförderung - ein Forschungsüberblick. Femina Politica - Zeitschrift für feministische Politikwissenschaft. 21(1): S. 158-162.

Schlegel, M. (2006). Professoren und Professorinnen an den Fachhochschulen in Niedersachsen - eine berufssoziologische empirische Untersuchung. Carl von Ossietzky Universität Oldenburg. Zugriff am 07.05.2020 unter http://oops.uni-oldenburg.de/750/1/schpro07.pdf.

Schmader, T., Whitehead J. \& Wysocki, V. (2007). A linguistic comparison of letters of recommendation for male and female chemistry and biochemistry job applicants. In: Sex Roles. 57: S. $509-514$

Schmidt, J. (2016). Entgeltgleichheit - Die gesamtwirtschaftliche Perspektive. Welche Ursachen hat der Gender Pay Gap? Köln: Institut der deutschen Wirtschaft.

Sembritzki, T. \& Thiele L. (2019). Geschlechterunterschiede bei Karrierewegen von Fachhochschulprofessorlnnen: eine empirische Bestandsaufnahme. In: Gender. Heft 1/ 2019: S. 11-30.

Sheridan, J., Pribbenow, C., Fine, E., Handelsman, J. \& Carnes, M. (2007). Climate Change at the University of Wisconsin-Madison: What changed, and did ADVANCE have an impact? Zugriff am 02.09.2016 unter: http://journals.psu.edu/wepan/article/viewFile/58488/58176.

Stark, S. \& Kiendl, A. (2013). Professorinnen - ubi estis? - wo seid Ihr? Bochum: Hochschule Bochum. Zugriff am 07.05.2020 unter https://www.h-brs.de/files/related/arbeitspapier _5_prof_innen_wo_seid_ihr.pdf

Statistisches Bundesamt (Destatis) (2019). Bildung und Kultur. Personal an Hochschulen. Wiesbaden: Statistisches Bundesamt. Zugriff am 07.05.2020 unter https://www.destatis.de/ GPStatistik/servlets/MCRFileNodeServlet/DEHeft_derivate_00054658/2110440187004.pdf

Steinpreis, R., Anders, K. \& Ritzke, D. (1999). The impact of gender on the review of the curricula vitae of job applicants and tenure candidates: A national empirical study. In: Sex Roles. 41: S. 409-528.

Steinweg, N. (2015). Geschlecht und Personalauswahl an Hochschulen und außerhochschulischen Forschungseinrichtungen. In: Peus, C., Braun, S., Hentschel, T., Frey, D. (Hrsg.). Personalauswahl in der Wissenschaft. Evidenzbasierte Methoden und Impulse für die Praxis. Heidelberg: Springer: S. 16-27.

van den Brink, M. (2015). Myths about Meritocracy and Transparency: The Role of Gender in Academic recruitment. In: Peus, C., Braun, S., Hentschel, T., Frey, D. (Hrsg.). Personalauswahl in der Wissenschaft. Evidenzbasierte Methoden und Impulse für die Praxis. Heidelberg: Springer: S. 191-201.

Wenneras, C. \& Wold, A. (1997). Nepotism and sexism in peer review. In: Nature. 387 (22): S. 341-343.

Winter, M. \& Krempkow, R. (2013). Kartierung der Hochschulforschung in Deutschland 2013. Bestandsaufnahme der hochschulforschenden Einrichtungen. Zugriff am 07.05.2020 unter: https://www.gfhf.net/wp-content/uploads/2014/09/Bericht-Kartierung-der-Hofo-2013.pdf

Wissenschaftsrat (Hrsg.) (2002). Empfehlungen zur Entwicklung der Fachhochschulen. Köln. Zugriff am 05.05.2020 unter https://www.wissenschaftsrat.de/download/archiv/5102-02.pdf; jsessionid=D407FD95EB7E37884646C9C4C9CC42CA.delivery2-master?_blob=publica tionFile \& $v=3$ 
Wissenschaftsrat (Hrsg.) (2016). Empfehlungen zur Gewinnung, Integration und Qualifizierung internationaler Studierender. Kiel. Zugriff am 05.05.2020 unter https://www.wissenschaftsrat.de/download/archiv/5437-16.pdf?__blob=publicationFile\&v=4

Wissenschaftsrat (Hrsg.) (2018). Empfehlungen zur Hochschulgovernance. Hannover. Zugriff am 05.05.2020 unter https://www.wissenschaftsrat.de/download/archiv/7328-18.pdf? blob=publicationFile $\& \mathrm{v}=15$

Zedlacher, E. \& Haas, M. (o.J.). Der Anpassungsdruck auf Frauen in Männerdomänen- Assimilationsstrategien und ihre Gefahren. Zugriff am 10.07.2018 unter: http://momentum-kon gress.org/cms/uploads/documents/Zedlacher_Haas_Abstract_Mom118_8_2011_0630.pdf 


\section{Bisher erschienene Bände der Schriftenreihe des GuTZ:}

Ausgabe 01/2009: Barthel, K. (Dez. 2009). Wer wagt, gewinnt? Geschlechtsspezifische Unterschiede im Entscheidungsverhalten unter Risiko.

https://www.beuth-hochschule.de/fileadmin/oe/gutz/Schriften reihe/GuTZ-Schriftenreihe_01_Dez2009_Barthel.pdf

Ausgabe 02/2010: Pattloch, A. (Dez. 2010). "Studieren in der Lounge" - Wie StudentInnen Ihre Hochschule gestalten würden. Eine geschlechterdifferenzierende Exploration.

https://www.beuth-hochschule.de/fileadmin/oe/gutz/Schriften reihe/GuTZ-Schriftenreihe_02_Dez2010_Pattloch.pdf

Ausgabe 03/2010: Ehmsen, S. (Dez. 2010). Die Vielfalt gestalten - Diversity an Hochschulen.

https://www.beuth-hochschule.de/fileadmin/oe/gutz/Schriften reihe/GuTZ-Schriftenreihe_03_Feb2010_Ehmsen.pdf

Ausgabe 04/2011: Gläsener, K. (April 2011). Diverse Teams = Erfolgsteams? Bedingungen für die Interaktion in geschlechts- und nationalitätsgemischten Teams. https://www.beuth-hochschule.de/fileadmin/oe/gutz/Schriften reihe/GuTZ-Schriftenreihe_04_Apr2011_Glaesener.pdf

Ausgabe 05/2012: Ihsen, S. und Ducki, A. (Juli 2012). Gender Toolbox. https://www.beuth-hochschule.de/fileadmin/oe/gutz/Schriften reihe/GuTZ-Schriftenreihe_05_Jul2012_Ihsen-Ducki_Gender_Toolbox.pdf

Ausgabe 06/2013: Erlemann, C. (Dez. 2013). Studentinnen mit Migrationshintergrund in Chancengleichheitsprojekten.

https://www.beuth-hochschule.de/fileadmin/oe/gutz/Schriften reihe/GuTZ-Schriftenreihe_06_Dez2013_Erlemann.pdf

Ausgabe 07/2014: Buchem, I. (Jan. 2014). Studentische Essays zum Thema Internet und Gesellschaft.

https://www.beuth-hochschule.de/fileadmin/oe/gutz/Schriften reihe/GuTZ-Schriftenreihe_07_Jan2014_Buchem.pdf

Ausgabe 08/2017: Brandt, M. und Ducki, A. (2017). Gendersensible Gestaltung des neuen Studiengangs „BWL - Digitale Wirtschaft“ an der Beuth Hochschule für Technik Berlin. Verlag Barbara Budrich, Leverkusen. https://shop.budrich-academic.de/produkt/gendersensible-gestaltungdes-neuen-studiengangs-bwl-digitale-wirtschaft-an-der-beuth-hochschule-fuer-technik-berlin/ 
Ausgabe 09/2018: Diallo-Ruschhaupt, U., Plaumann, S. und Dombrowski, E.-M. (2018). Karrierewege zu einer Professur an einer Fachhochschule. Verlag Barbara Budrich, Leverkusen.

https://shop.budrich-academic.de/produkt/karrierewege-zur-professuran-einer-fachhochschule/

Ausgabe 10/2019: $\quad$ Gläsener, K.,Afflerbach, T. und Ducki, A. (2019). Design Thinking, Digi talisierung und Diversity Management. Ein Praxisleifaden für die Lehre. Verlag Barbara Budrich, Leverkusen.

https://shop.budrich-academic.de/produkt/design-thinking-digitalisier ung-und-diversity-management/ 


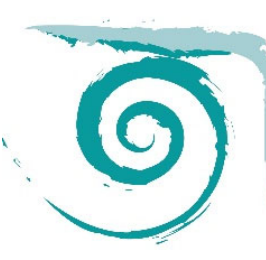

\section{GuTZ \\ GENDER- UND \\ TECHNIK-ZENTRUM}

Die Partizipation von Frauen an Professuren ist in den letzten Jahren gestiegen, dennoch sind Professorinnen im Wissenschaftssystem nach wie vor unterrepräsentiert. Studien, insbesondere über die Karrierewege von Professorinnen an technischen Hochschulen, sind selten, Ergebnisse entsprechend wenig evidenzbasiert. An der Beuth Hochschule für Technik Berlin wurde für die vorliegende Studie nun eine Organisationsklimaanalyse durchgeführt und geschlechterdifferenziert ausgewertet. Auch wenn die Studie aufgrund der kleinen Stichprobe nicht repräsentativ ist, hat sie zu aufschlussreichen Ergebnissen und ersten Anregungen geführt, wie geschlechterspezifische Diskriminierung abgebaut und eine geschlechtergerechte Hochschulorganisation aufgebaut werden kann.

\section{Schriftenreihe des Gender- und Technik-Zentrums der Beuth Hochschule für Technik Berlin}

Herausgeberinnen:

Prof. in Dr. in Eva-Maria Dombrowsk eva-maria.dombrowski@beuth-hochschule.de

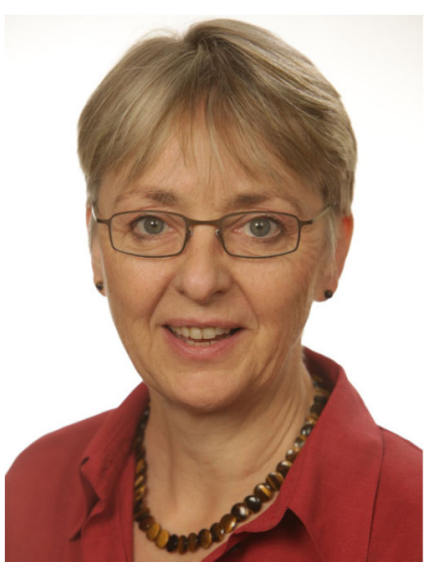

Prof. in Dr. ${ }^{\text {in }}$ Antje Ducki ducki@beuth-hochschule.de

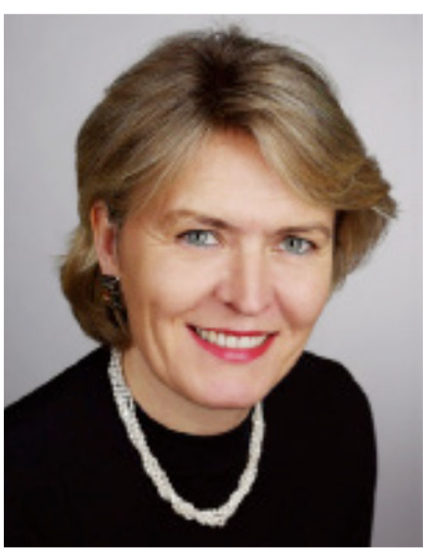

